\title{
Block Diagonal Dominance-based Model Reduction Method Applied to MMC Asymmetric Stability Analysis
}

\author{
Zong, Haoxiang; Zhang, Chen; Lyu, Jing; Cai, Xu; Molinas, Marta
}

Published in:

IEEE Transactions on Energy Conversion

Link to article, DOI:

10.1109/TEC.2021.3054925

Publication date:

2021

Document Version

Peer reviewed version

Link back to DTU Orbit

Citation (APA):

Zong, H., Zhang, C., Lyu, J., Cai, X., \& Molinas, M. (2021). Block Diagonal Dominance-based Model Reduction Method Applied to MMC Asymmetric Stability Analysis. IEEE Transactions on Energy Conversion, 36(3), 2438 2451. https://doi.org/10.1109/TEC.2021.3054925

\section{General rights}

Copyright and moral rights for the publications made accessible in the public portal are retained by the authors and/or other copyright owners and it is a condition of accessing publications that users recognise and abide by the legal requirements associated with these rights.

- Users may download and print one copy of any publication from the public portal for the purpose of private study or research.

- You may not further distribute the material or use it for any profit-making activity or commercial gain

- You may freely distribute the URL identifying the publication in the public portal 


\title{
Block Diagonal Dominance-based Model Reduction Method Applied to MMC Asymmetric Stability Analysis
}

\author{
Haoxiang Zong, Student Member, IEEE, Chen Zhang, Member, IEEE, Jing Lyu, Senior Member, \\ IEEE, Xu Cai, Marta Molinas, Member, IEEE
}

\begin{abstract}
Frequency-domain model reduction is a crucial concern in applying the prevailing impedance method for the stability analysis of complex systems, e.g., the modular multilevel converter (MMC). Recently, it has been shown that under symmetric conditions, a $2 \times 2$ matrix-based impedance model characterizing the two coupled frequencies of MMC are sufficient for its stability analysis. However, when the asymmetry occurs, principally, a much higher number of frequency couplings will appear in the MMC and thereby leads to a significant rise in the model dimension. Enlighted by this issue, there is an urgent need of finding a suitable frequency-domain method that can serve as a general criterion for model reduction. To this end, this paper proposes a block diagonal dominance (BDD)-based model reduction method and applied it to the asymmetric MMC. Basically, the BDD can decompose an $N$-dimensional task to $N$ one-dimensional tasks, via which a significant reduction in model dimension can be realized. It is shown that by properly shifting the impedance model from one domain to another (e.g., $\alpha-\beta$ domain to $d-q$ domain), the BDD property can be achieved for most asymmetric scenarios. Finally, various case studies considering different asymmetry degrees are conducted to validate the effectiveness of the proposed method.
\end{abstract}

Index Terms-MMC, asymmetric, reduced-order model, block diagonal dominance (BDD), impedance, stability

\section{INTRODUCTION}

$M$ ODULAR Multilevel Converter (MMC), with its inherent advantages such as built-in redundancy, higher efficiency, and lower harmonics, has gained its popularity in applications of high voltage direct current (HVDC) transmissions [1], [2]. However, recent practices have shown that serious small-signal instability problem [3] may occur due to control interaction, which poses significant challenges for the system's stable and reliable operation.

In this respect, numerous studies aimed at characterizing small-signal behaviors of the MMC and associated stability analysis have been recently carried out, where the adopted methods mainly include dynamic phasor [4]-[6], harmonic

This work was supported in part by the National Natural Science Foundation of China under Grant 51837007 and in part by the China Scholarship Council under Grant 201906230208.

Haoxiang Zong, Jing Lyu and Xu Cai are with the Key Laboratory of Control of Power Transmission and Conversion of Ministry of Education, Shanghai Jiao Tong University, Shanghai, China. (e-mail: haoxiangzong@sjtu.edu.cn). state-space (HSS) [7], harmonic linearization [8], etc. However, most of them are focused on the three-phase symmetric operating states, while the asymmetric operating states have not been paid much attention. Typical asymmetric issues [9] of the MMC family include discrepancy of branch inductor parameters, asymmetry of branch submodules (SM) numbers in case of SM failures, inequality of capacitor voltage, dynamic load condition, unbalanced ac-grid condition, etc. Under asymmetric conditions, the internal dynamics of the MMC will become much more complicated, because of current waveform distortion, voltage ripple aggravation and multi-frequency couplings [10]-[12], etc. All these factors pose a significant challenge for the asymmetric stability analysis, however, so far, very few related studies have been devoted to this issue. Refs [13], [14] mainly analyzed the effect of control parameters on the stability of the MMC under asymmetric conditions, both of which were based on the state-space model developed by the dynamic phasor. Despite that Refs [13], [14] have gained valuable insights into the potential stability issues of the MMC arising from three-phase asymmetries, more dedicated analyses are deserved, e.g., from the impedance perspective.

To the best knowledge of the authors, the impedance-based asymmetric stability analysis of the MMC has so far been missing, where the main difficulty may lie in the applicability of the resulting high-dimensional impedance to practical analysis. Specifically, for symmetric cases, as demonstrated in [15]-[17], a $2 \times 2$ impedance matrix can accurately depict the MMC AC-side dynamics, because there mainly exists one pair of frequency couplings. However, for asymmetric cases, multiple frequency couplings will appear, especially those at the same frequency (denoted by the sequence coupling [8]). Consequently, the dimension of the impedance matrix will be much larger than that of the $2 \times 2$ matrix developed for symmetric cases, i.e., the resulting impedance model will be a high-dimensional multi-input-multi-output (MIMO) system. Although the Generalized Nyquist Criterion (GNC) is in principle applicable to impedance matrices regardless of

Chen Zhang is with Department of Electrical Engineering, Technical University of Denmark, Copenhagen, Denmark. (e-mail: chezh@elektro.dtu.dk).

Marta Molinas is with Department of Engineering Cybernetics, Norwegian University of Science and Technology, Trondheim, Norway. (e-mail: marta.molinas@ntnu.no).

Corresponding author: Chen Zhang; Xu Cai 
dimensions, the resulting multiple Nyquist diagrams will be difficult to be inspected and interpreted. More importantly, using such a high-dimensional model will be challenging for interconnected stability analysis, especially connected with low-dimensional impedance models. Therefore, in the presence of three-phase asymmetries, a reduced-order impedance model will be beneficial for simplifying the stability analysis of the MMC or various MMC-based transmission systems.

In this regard, mainly two model reduction methods [18] for the MIMO system can be considered: (1) transforming the $\mathrm{N}$ dimensional MIMO system into the equivalent 1-dimensional form; (2) reducing the $\mathrm{N}$-dimensional MIMO system into a set of separate single-loop forms. The first method originated from the individual channel design theory [18], now so-called singleinput-single-output (SISO) model, which can preserve all information of the high-dimensional MIMO system. However, it is, in fact, a closed-loop model dependent on both the source and load systems [19], [20]. Therefore, the nonexistence of right-half-plane (RHP) poles cannot be assured and should be re-evaluated which brings inconveniences to the stability analysis. The second method is based upon a careful use of a specific criterion of partial interaction, the diagonal dominance concept [21]. The latter method is more preferred in the interconnected analysis, because it is aimed at the open-loop model which has many good properties like no RHPs, more flexibility, etc.

In fact, not all coupling elements within the high-dimensional MIMO impedance matrix will have significant influence on the stability margin, which means those with weak strength can be discarded. This qualitative understanding implies that there exists a headroom for the model reduction. However, the main difficulties lie in the proposition of a feasible reduction criterion. To bridge this gap, based on the block diagonal dominance (BDD) theory [21]-[23], this paper proposes a model reduction method for MMC asymmetric stability analysis. It is noted that the obtained reduced-order model will not compromise the accuracy of stability judgements for most asymmetric scenarios, which is a major merit of this method.

The rest of the paper is arranged as follows. In Section II, the MIMO impedance model of the MMC is introduced, based on which a qualitative analysis of the asymmetric effects is carried out; In Section III, according to the BDD theory, the model reduction method is presented, and the reduced-order impedance model is obtained; In Section IV, asymmetric branch conditions and asymmetric ac-grid conditions are used to test the effectiveness of the proposed method; Section V concludes the paper.

\section{MIMO IMPEDANCE MODEL OF THE MMC AND ANALYSIS OF ASYMMETRIC EFFECTS}

In this section, multi-frequency couplings within the MMC under asymmetric conditions are studied qualitatively and the MIMO impedance model along with its verification is given. Then, the existing difficulties for the impedance-based asymmetric stability analysis are illustrated, which motivates the necessity of model reduction discussed in the next section.

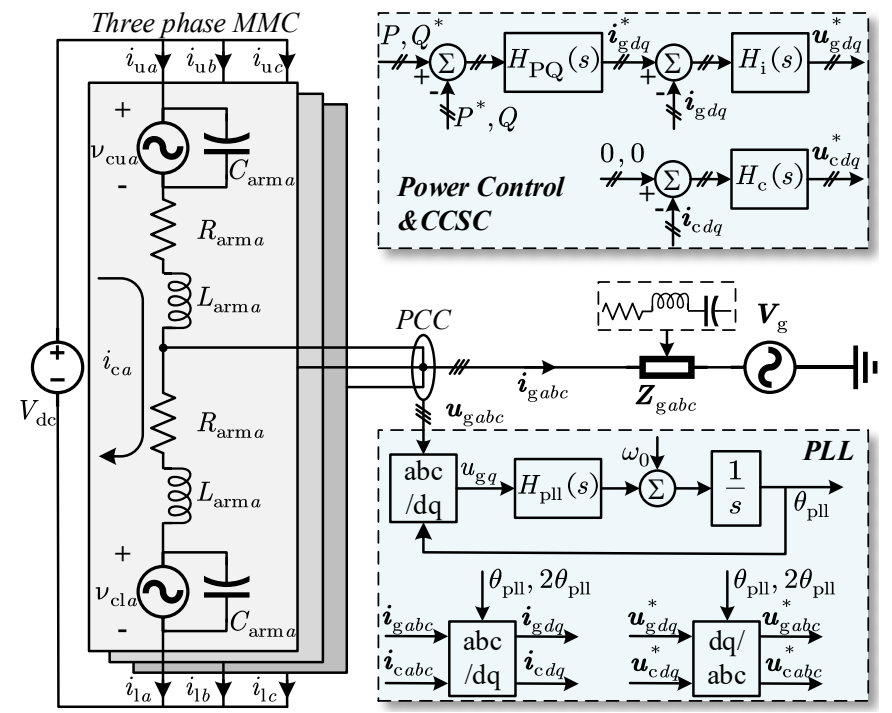

Fig. 1. The three-phase grid-tied MMC system

\section{A. Qualitative Analysis of the Asymmetric Effects}

Fig. 1 presents a typical grid-tied MMC system, which is mainly composed of a three-phase MMC and a Thevenin equivalent $\mathrm{AC}$ grid. The $\mathrm{DC}$ voltage is assumed constant in this study, whereas the power control, circulating current suppressing control (CCSC) and the phase locked loop (PLL) will be modelled in detail.

To first acquire a qualitative cognition of the MMC smallsignal characteristics under symmetric and asymmetric conditions, a small-signal voltage perturbation of $25 \mathrm{~Hz}$ is injected at the point of common coupling (PCC) and the frequency response of the PCC current is shown in Fig. 2. For symmetric conditions, it can be seen from Fig. 2(a) that only one pair of frequency couplings $(25 \mathrm{~Hz} \& 75 \mathrm{~Hz})$ appears, the coupling mechanism of which has been studied well in [15][17]. Refs [15]-[17] also explain why a $2 \times 2$ impedance matrix is adequately accurate for reflecting the MMC AC-side dynamics. For asymmetric conditions $\left(L_{\mathrm{arm}}=L_{\mathrm{arm} c}=0.36 \mathrm{H}\right.$, $L_{\mathrm{arm} a}=3 \times 0.36 \mathrm{H}$ ), it can be seen from Fig. 2(b) that more than one pair of frequency couplings are generated in the feedback current, such as $25 \mathrm{~Hz}, 75 \mathrm{~Hz}, 125 \mathrm{~Hz}$ and $175 \mathrm{~Hz}$. Moreover, three-phase harmonic components of each coupled frequency are different from each other, which means the sequence coupling cannot be ignored at this time. To consider these multi-frequency couplings, a high-dimensional MIMO impedance matrix is needed.

\section{B. MIMO Impedance Model of the MMC}

Irrespective of being under symmetric or asymmetric conditions, the MMC is always a nonlinear time-periodically

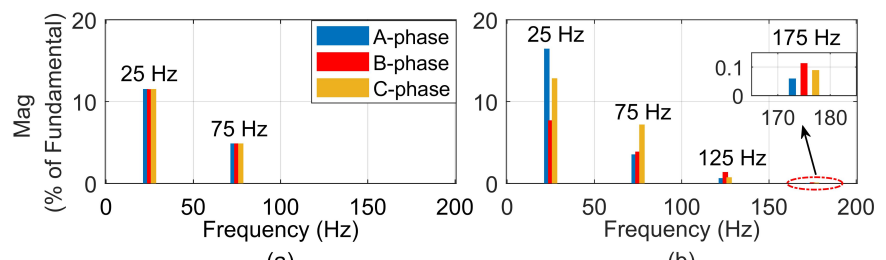

Fig. 2. FFT analysis of the PCC current, (a) symmetric; (b) asymmetric 
varying system, which can be modelled via the HSS method. In this part, a $14 \times 14$ MIMO impedance matrix of the MMC is established [7], [24], where the modelling process of the main circuit, power control and CCSC is presented in detail.

\section{1) HSS Model of the Main Circuit}

The HSS theory has been amply discussed recently [15]-[20], and thus not presented here for brevity. The averaged dynamic model of the MMC is adopted and the corresponding HSS equations of the main circuit are given in (1). In what follows, boldface characters refer to vector or matrix quantities.

$$
\boldsymbol{N}_{\mathrm{hss}} \underbrace{\left[\begin{array}{c}
\boldsymbol{\Delta} \boldsymbol{i}_{\mathrm{c} \boldsymbol{x}}^{\mathrm{hss}} \\
\boldsymbol{\Delta} \boldsymbol{\nu}_{\mathrm{cu} \boldsymbol{x}}^{\mathrm{hss}} \\
\boldsymbol{\Delta} \boldsymbol{\nu}_{\mathrm{cl} \boldsymbol{x}}^{\mathrm{hss}} \\
\boldsymbol{\Delta} \boldsymbol{i}_{\mathrm{g} \boldsymbol{x}}^{\mathrm{hss}}
\end{array}\right]}_{\boldsymbol{X}_{\mathrm{hss}}}=\boldsymbol{a}_{\mathrm{hss}} \boldsymbol{X}_{\mathrm{hss}}+\boldsymbol{m}_{\mathrm{hss}}\left[\begin{array}{c}
\boldsymbol{\Delta} \boldsymbol{m}_{\mathrm{u} \boldsymbol{x}}^{\mathrm{hs}} \\
\boldsymbol{\Delta} \boldsymbol{m}_{\mathrm{l} \boldsymbol{x}}^{\mathrm{hss}}
\end{array}\right]+\boldsymbol{b}_{\mathrm{hss}} \underbrace{\left[\begin{array}{c}
\boldsymbol{\Delta} \boldsymbol{u}_{\mathrm{g} \boldsymbol{x}}^{\mathrm{hss}} \\
0 \\
0 \\
0
\end{array}\right]}_{\boldsymbol{U}_{\mathrm{hss}}}
$$

where the $\boldsymbol{X}_{\mathrm{hss}}$ is the state-vector, consisting of the $\Delta \boldsymbol{\Delta i}_{\mathrm{c} x}^{\mathrm{hss}}, \boldsymbol{\Delta} \boldsymbol{v}_{\mathrm{cux}}^{\mathrm{hs}}$, $\Delta \boldsymbol{v}_{\mathrm{cl} x}^{\mathrm{hs}}, \boldsymbol{\Delta i _ { \mathrm { g } x } ^ { \mathrm { hss } }}(\boldsymbol{x}=a, b, c)$, which represent the three-phase HSS form of the circulating current, upper arm voltage, lower arm voltage and PCC current, respectively. The $\boldsymbol{U}_{\text {hss }}$ is the inputvector, consisting of $\Delta u_{\mathrm{g} x}^{\mathrm{hss}}$ which represents the three-phase HSS form PCC voltage. The $\boldsymbol{N}_{\mathrm{hss}}, \boldsymbol{a}_{\mathrm{hss}}, \boldsymbol{m}_{\mathrm{hss}}$ and $\boldsymbol{b}_{\mathrm{hss}}$ are all Toeplitz matrices as defined in Appendix A.

The concrete expression of $\Delta \boldsymbol{i}_{\mathrm{g} x}^{\mathrm{hss}}$ is given in (2) as an example, for better illustration of the symbol definition.

$$
\boldsymbol{\Delta} i_{\mathrm{g} x}^{\mathrm{hss}}=\left[\begin{array}{c}
\boldsymbol{\Delta} i_{\mathrm{g} a}^{\mathrm{hss}} \\
\boldsymbol{\Delta} i_{\mathrm{g} b}^{\mathrm{hss}} \\
\boldsymbol{\Delta} i_{\mathrm{g} c}^{\mathrm{hss}}
\end{array}\right], \quad \boldsymbol{\Delta} i_{\mathrm{g} a}^{\mathrm{hss}}=\left[\Delta i_{\mathrm{g} a}^{-h}, \cdots, \Delta i_{\mathrm{g} a}^{0}, \cdots, \Delta i_{\mathrm{g} a}^{h}\right]^{T}
$$

where the superscript ' $h$ ' represents the considered harmonics order in the HSS model.

The modulation ratio $\Delta m_{\mathrm{u} x}^{\mathrm{hs}}, \Delta m_{\mathrm{l} x}^{\mathrm{hs}}$ can be expressed as:

$$
\left[\begin{array}{c}
\boldsymbol{\Delta} \boldsymbol{m}_{\mathrm{u} \boldsymbol{x}}^{\mathrm{hss}} \\
\boldsymbol{\Delta} \boldsymbol{m}_{\mathrm{l} \boldsymbol{x}}^{\mathrm{hss}}
\end{array}\right]=\frac{1}{V_{\mathrm{dc}}}\left[\begin{array}{l}
-\boldsymbol{\Delta} \boldsymbol{u}_{\mathrm{c} \boldsymbol{x}}^{\mathrm{hs} *}-\boldsymbol{\Delta} \boldsymbol{u}_{\mathrm{g} \boldsymbol{x}}^{\mathrm{hs*}} \\
-\boldsymbol{\Delta} \boldsymbol{u}_{\mathrm{c} \boldsymbol{x}}^{\mathrm{hs} *}+\boldsymbol{\Delta} \boldsymbol{u}_{\mathrm{g} \boldsymbol{x}}^{\mathrm{hs} *}
\end{array}\right]
$$

where the $\Delta u_{\mathrm{g} x}^{\mathrm{hs}^{*}}$ and $\Delta u_{\mathrm{cx}}^{\mathrm{hs*}}$ represent the output modulation voltages of the power control and CCSC, respectively.

2) HSS Model of the Power Control

At first, the HSS model of the Park transformation is given in (4), which is essentially a memoryless linear time periodically varying (LTPV) system [24].

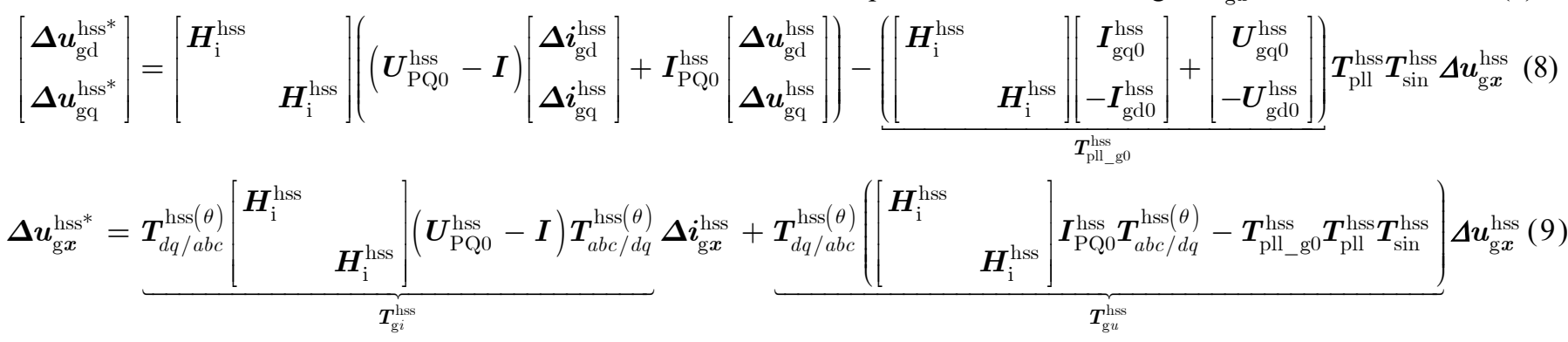

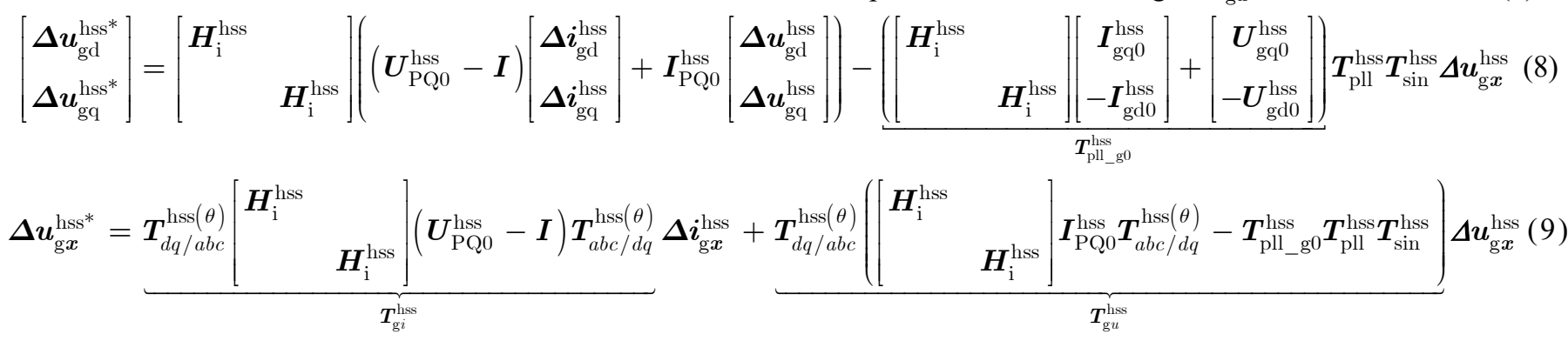

where the submatrices like $\boldsymbol{A}_{\mathrm{cos} b}^{\mathrm{hss}}$ or $\boldsymbol{A}_{\mathrm{cos} 2 b}^{\mathrm{hss}}$ are the Toeplitz matrices of $\cos \left(\omega_{1} t-2 \pi / 3\right)$ and $\cos \left(2 \omega_{1} t+2 \pi / 3\right)$. For example, the nonzero elements of $\boldsymbol{A}_{\text {cos } b}^{\text {hss }}$ are $A_{+1}=a^{*} / 2$, $A_{-1}=a / 2$ with $a=e^{j 2 \pi / 3}$.

The HSS model of the phase locked loop (PLL) is like:

$$
\Delta \theta_{\mathrm{pll}}^{\mathrm{hss}}=\underbrace{\frac{\boldsymbol{H}_{\mathrm{pll}}^{\mathrm{hss}}}{s \boldsymbol{I}+U_{\mathrm{pl}}^{\mathrm{hss}} \cdot H_{\mathrm{pll}}^{\mathrm{hss}}}}_{T_{\mathrm{pll}}^{\mathrm{hss}}} T_{\mathrm{sin}}^{\mathrm{hss}} \cdot \Delta u_{\mathrm{g} \boldsymbol{x}}^{\mathrm{hss}}
$$

where $\quad \boldsymbol{H}_{\mathrm{pll}}^{\mathrm{hss}}=\operatorname{diag}\left(\ldots, H_{\mathrm{pll}}\left(s-j \omega_{1}\right), H_{\mathrm{pll}}(s), H_{\mathrm{pll}}\left(s+j \omega_{1}\right), \ldots\right)$ are obtained via frequency shifting of the $H_{\mathrm{pll}}(s)=k_{\mathrm{p}}^{\mathrm{pll}}+k_{\mathrm{i}}^{\mathrm{pll}} / s$. The operation point $\boldsymbol{U}_{\mathrm{gd} 0}^{\mathrm{hss}}$ is a Toeplitz matrix same as the ' $\boldsymbol{U}_{\mathrm{gd}}^{\mathrm{htf}}$, defined in [24].

The HSS model of the PQ outer loop is like:

$$
\begin{aligned}
& {\left[\begin{array}{c}
\Delta i_{\mathrm{gd}}^{\mathrm{hs*}} \\
\Delta \boldsymbol{i}_{\mathrm{gq}}^{\mathrm{hss}}
\end{array}\right]=\underbrace{\frac{3}{2}\left[\begin{array}{rr}
\boldsymbol{H}_{\mathrm{PQ}}^{\mathrm{hss}} & \\
& -\boldsymbol{H}_{\mathrm{PQ}}^{\mathrm{hss}}
\end{array}\right]\left[\begin{array}{cc}
\boldsymbol{U}_{\mathrm{gd} 0}^{\mathrm{hss}} & \boldsymbol{U}_{\mathrm{gq} 0}^{\mathrm{hss}} \\
-\boldsymbol{U}_{\mathrm{gq} 0}^{\mathrm{hss}} & \boldsymbol{U}_{\mathrm{gd} 0}^{\mathrm{hss}}
\end{array}\right]}_{\boldsymbol{U}_{\mathrm{PO} 0}^{\mathrm{hs}}} \cdot\left[\begin{array}{c}
\Delta \boldsymbol{i}_{\mathrm{gd}}^{\mathrm{hss}} \\
\Delta \boldsymbol{i}_{\mathrm{gq}}^{\mathrm{hss}}
\end{array}\right]}
\end{aligned}
$$

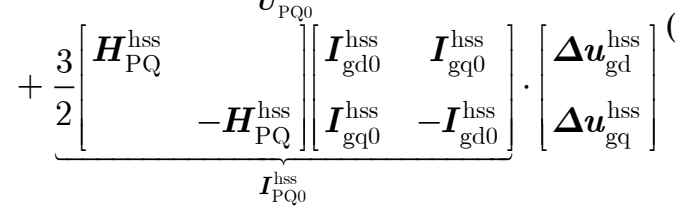

where $\boldsymbol{H}_{\mathrm{PQ}}^{\mathrm{hss}}$ are the frequency-shifted copies of the $H_{\mathrm{PQ}}(s)=$ $k_{\mathrm{p}}^{\mathrm{PQ}}+k_{\mathrm{i}}^{\mathrm{PQ}} / s$. The $\boldsymbol{U}_{\mathrm{gq} 0}^{\mathrm{hss}}, \boldsymbol{I}_{\mathrm{gd} 0}^{\mathrm{hss}}$ and $\boldsymbol{I}_{\mathrm{gq} 0}^{\mathrm{hss}}$ are all Toeplitz matrices.

The HSS model of the PQ inner loop considering the PLL dynamics is given in (7).

$$
\begin{aligned}
& {\left[\begin{array}{c}
\Delta \boldsymbol{u}_{\mathrm{gd}}^{\mathrm{hss}^{*}} \\
\boldsymbol{\Delta} \boldsymbol{u}_{\mathrm{gq}}^{\mathrm{hss}}
\end{array}\right]+\left[\begin{array}{c}
\boldsymbol{U}_{\mathrm{gq} 0}^{\mathrm{hss}} \\
-\boldsymbol{U}_{\mathrm{gd}}^{\mathrm{hss}}
\end{array}\right] \boldsymbol{\Delta} \boldsymbol{\theta}_{\mathrm{pll}}^{\mathrm{hss}}} \\
& =\left[\begin{array}{ll}
\boldsymbol{H}_{\mathrm{i}}^{\mathrm{hss}} & \\
& \boldsymbol{H}_{\mathrm{i}}^{\mathrm{hss}}
\end{array}\right]\left(\left[\begin{array}{c}
\boldsymbol{\Delta} i_{\mathrm{gd}}^{\mathrm{hs} *} \\
\boldsymbol{\Delta} i_{\mathrm{gq}}^{\mathrm{hs}}
\end{array}\right]-\left[\begin{array}{c}
\boldsymbol{\Delta} i_{\mathrm{gd}}^{\mathrm{hss}} \\
\boldsymbol{\Delta} i_{\mathrm{gq}}^{\mathrm{hss}}
\end{array}\right]-\left[\begin{array}{c}
\boldsymbol{I}_{\mathrm{gq} 0}^{\mathrm{hss}} \\
-\boldsymbol{I}_{\mathrm{gd} 0}^{\mathrm{hss}}
\end{array}\right] \boldsymbol{\Delta} \boldsymbol{\theta}_{\mathrm{pll}}^{\mathrm{hss}}\right)
\end{aligned}
$$

where $\boldsymbol{H}_{\mathrm{i}}^{\mathrm{hss}}$ are the frequency-shifted copies of the $H_{\mathrm{i}}(s)=$ $k_{\mathrm{pi}}+k_{\mathrm{ii}} / s$.

Substituting (5) and (6) into (7) yields (8), and by applying the Park transformation at fundamental frequency to (8), the three-phase modulation voltage $\Delta u_{\mathrm{g} x}^{\mathrm{hss}^{*}}$ can be obtained in (9). 


\section{3) HSS Model of the CCSC}

The HSS model of CCSC with the PLL dynamics is like:

$$
\begin{aligned}
{\left[\begin{array}{c}
\boldsymbol{\Delta} \boldsymbol{u}_{\mathrm{cd}}^{\mathrm{hs} *} \\
\boldsymbol{\Delta} \boldsymbol{u}_{\mathrm{cq}}^{\mathrm{hs} *}
\end{array}\right]=\left[\begin{array}{rr}
\boldsymbol{H}_{\mathrm{c}}^{\mathrm{hss}} & \\
& \boldsymbol{H}_{\mathrm{c}}^{\mathrm{hss}}
\end{array}\right]\left(\left[\begin{array}{l}
\mathbf{0} \\
\mathbf{0}
\end{array}\right]-\left[\begin{array}{c}
\boldsymbol{\Delta} \boldsymbol{i}_{\mathrm{gd}}^{\mathrm{hs}} \\
\boldsymbol{\Delta} \boldsymbol{i}_{\mathrm{gq}}^{\mathrm{hss}}
\end{array}\right]\right) } \\
\left.-\left(\begin{array}{rr}
\boldsymbol{H}_{\mathrm{c}}^{\mathrm{hss}} & \\
& \boldsymbol{H}_{\mathrm{c}}^{\mathrm{hss}}
\end{array}\right]\left[\begin{array}{c}
-\boldsymbol{I}_{\mathrm{cq} 0}^{\mathrm{hss}} \\
\boldsymbol{I}_{\mathrm{cd} 0}^{\mathrm{hss}}
\end{array}\right]+\left[\begin{array}{c}
-\boldsymbol{U}_{\mathrm{cq} 0}^{\mathrm{hs}} \\
\boldsymbol{U}_{\mathrm{cd} 0}^{\mathrm{hss}}
\end{array}\right]\right) \cdot 2 \boldsymbol{\Delta} \boldsymbol{\theta}_{\mathrm{pll}}^{\mathrm{hss}}
\end{aligned}
$$

where $\boldsymbol{H}_{\mathrm{c}}^{\mathrm{hss}}$ are the frequency-shifted copies of the $H_{\mathrm{c}}(s)=$ $k_{\mathrm{pc}}+k_{\mathrm{ic}} / s$. The $\boldsymbol{U}_{\mathrm{cd} 0}^{\mathrm{hss}}, \boldsymbol{U}_{\mathrm{cq} 0}^{\mathrm{hss}}, \boldsymbol{I}_{\mathrm{cd} 0}^{\mathrm{hss}}$ and $\boldsymbol{I}_{\mathrm{cq} 0}^{\mathrm{hss}}$ are Toeplitz matrices.

The three-phase output modulation voltage $\Delta \boldsymbol{u}_{\mathrm{c} x}^{\mathrm{hss}^{*}}$ is obtained in (11) by substituting (4) and (5) into (10).

$$
\begin{aligned}
& \boldsymbol{\Delta} \boldsymbol{u}_{\mathrm{c} \boldsymbol{x}}^{\mathrm{hs} *^{*}}=\underbrace{-\boldsymbol{T}_{d q / a b c}^{\mathrm{hs}(2 \theta)}\left[\boldsymbol{H}_{\mathrm{c}}^{\mathrm{hss}} \boldsymbol{H}_{\mathrm{c}}^{\mathrm{hss}}\right] \boldsymbol{T}_{a b c / d q}^{\mathrm{hss}(2 \theta)}}_{\boldsymbol{T}_{\mathrm{c} i}^{\mathrm{hss}}} \cdot \boldsymbol{\Delta} \boldsymbol{i}_{\mathrm{c} \boldsymbol{x}}^{\mathrm{hss}} \\
& +\underbrace{-2 \boldsymbol{T}_{d q / a b c}^{\mathrm{hss}(2 \theta)} \boldsymbol{T}_{\mathrm{pll}}^{\mathrm{hss} \mathrm{c} 0} \boldsymbol{T}_{\mathrm{pll}}^{\mathrm{hss}} \boldsymbol{T}_{\mathrm{sin}}^{\mathrm{hss}}}_{\boldsymbol{T}_{\mathrm{c} u}^{\mathrm{hss}}} \cdot \boldsymbol{\Delta} \boldsymbol{u}_{\mathrm{g} \boldsymbol{x}}^{\mathrm{hss}}
\end{aligned}
$$

\section{4) MIMO Impedance Model}

By substituting (9) and (11) into (3), the modulation ratio $\Delta m_{u x}^{\text {hss }}, \Delta m_{l x}^{\text {hss }}$ can be represented totally by the state variables $\Delta i_{\mathrm{c} x}^{\mathrm{hss}}, \boldsymbol{\Delta} \boldsymbol{i}_{\mathrm{g} x}^{\mathrm{hss}}$ and input variables $\boldsymbol{\Delta} \boldsymbol{u}_{\mathrm{g} x}^{\mathrm{hss}}$ as:

$$
\left[\begin{array}{c}
\Delta \boldsymbol{m}_{\mathrm{u} x}^{\mathrm{hss}} \\
\Delta \boldsymbol{m}_{\mathrm{l} \boldsymbol{x}}^{\mathrm{hss}}
\end{array}\right]=\frac{-1}{V_{\mathrm{dc}}}\left[\begin{array}{ccc}
\boldsymbol{T}_{\mathrm{c} i}^{\mathrm{hss}} & \boldsymbol{T}_{\mathrm{g} i}^{\mathrm{hss}} & \boldsymbol{T}_{\mathrm{g} u}^{\mathrm{hss}}+\boldsymbol{T}_{\mathrm{c} u}^{\mathrm{hss}} \\
\boldsymbol{T}_{\mathrm{c} i}^{\mathrm{hss}} & -\boldsymbol{T}_{\mathrm{g} i}^{\mathrm{hss}} & \boldsymbol{T}_{\mathrm{c} u}^{\mathrm{hss}}-\boldsymbol{T}_{\mathrm{g} u}^{\mathrm{hss}}
\end{array}\right]\left[\begin{array}{c}
\boldsymbol{\Delta} \boldsymbol{i}_{\mathrm{c} x}^{\mathrm{hss}} \\
\boldsymbol{\Delta} \boldsymbol{i}_{\mathrm{g} x}^{\mathrm{hss}} \\
\boldsymbol{\Delta} \boldsymbol{u}_{\mathrm{g} \boldsymbol{x}}^{\mathrm{hss}}
\end{array}\right]
$$

Substituting (12) into (1) yields the HSS model of the MMC:

$$
\boldsymbol{X}_{\mathrm{hss}}=\underbrace{-\left(\boldsymbol{A}_{\mathrm{hss}}-\boldsymbol{N}_{\mathrm{hss}}\right)^{-1} \boldsymbol{B}_{\mathrm{hss}}}_{\chi_{\mathrm{hss}}} \cdot \boldsymbol{U}_{\mathrm{hss}}
$$

where the definition of $\boldsymbol{A}_{\mathrm{hss}}$ and $\boldsymbol{B}_{\mathrm{hss}}$ is given in Appendix A.

By expanding the (13) in matrix form, the AC-side admittance, i.e., the input-output relationship between the PCC voltage $\Delta u_{\mathrm{g} x}^{\mathrm{hss}}$ and the PCC current $\Delta \mathrm{i}_{\mathrm{g} x}^{\mathrm{hss}}$, can be extracted as:

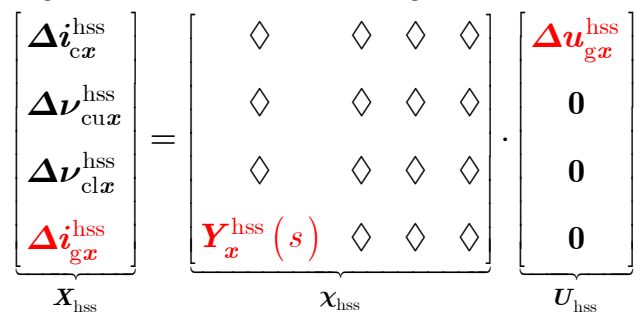

Usually, the accuracy of the HSS model can be ensured by setting $h=3$ [7]. The admittance $\boldsymbol{Y}_{x}^{\mathrm{hss}}(s)$ is transformed into the impedance and rearranged in harmonic order as:

$$
\left[\begin{array}{c}
\Delta \boldsymbol{u}_{\mathrm{g} \boldsymbol{x}}^{-3 \omega_{1}} \\
\vdots \\
\Delta \boldsymbol{u}_{\mathrm{g} \boldsymbol{x}}^{+3 \omega_{1}}
\end{array}\right]=\boldsymbol{Z}_{\boldsymbol{x}}^{\mathrm{mmc}}(s)_{21 \times 21} \cdot\left[\begin{array}{c}
\Delta \boldsymbol{i}_{\mathrm{g} \boldsymbol{x}}^{-3 \omega_{1}} \\
\vdots \\
\Delta \boldsymbol{i}_{\mathrm{g} \boldsymbol{x}}^{+3 \omega_{1}}
\end{array}\right]
$$

where for example, $\Delta i_{\mathrm{g} x}^{-3 \omega_{1}}=\left[\begin{array}{lll}\Delta i_{\mathrm{g} a}^{-3 \omega_{1}} & \Delta i_{\mathrm{g} b}^{-3 \omega_{1}} & \Delta i_{\mathrm{gc}}^{-3 \omega_{1}}\end{array}\right]^{T}$.

Since zero-sequence components usually have no flow path in MMC [6], the three-phase system can be represented by a two-phase system $(\alpha-\beta)$ through the Clarke transformation, then using the vector representation of variables in $\alpha-\beta$ frame, i.e., $\alpha+j \beta$ and $\alpha-j \beta$. The following relationship can be established:

$$
\left[\begin{array}{c}
u_{\alpha \beta+}^{h \omega_{1}} \\
u_{\alpha \beta-}^{h \omega_{1}}
\end{array}\right]=\left[\begin{array}{c}
u_{\alpha}^{h \omega_{1}}+j u_{\beta}^{h \omega_{1}} \\
u_{\alpha}^{h \omega_{1}}-j u_{\beta}^{h \omega_{1}}
\end{array}\right]=\underbrace{\frac{2}{3}\left[\begin{array}{ccc}
1 & e^{j \frac{2}{3} \pi} & e^{j \frac{4}{3} \pi} \\
1 & e^{j \frac{4}{3} \pi} & e^{j-\frac{2}{3} \pi}
\end{array}\right]}_{T_{a b c / \alpha \beta \pm}} \cdot\left[\begin{array}{l}
u_{a}^{h \omega_{1}} \\
u_{b}^{h \omega_{1}} \\
u_{c}^{h \omega_{1}}
\end{array}\right]
$$

Based on the (16), the MIMO impedance in $\alpha-\beta$ domain can be calculated from the one developed in the $a b c$-frame as:

$$
Z_{\alpha \beta \pm}^{\mathrm{mmc}}(s)=\boldsymbol{T}_{a b c / \alpha \beta \pm}^{\mathrm{hss}} \cdot \boldsymbol{Z}_{\boldsymbol{x}}^{\mathrm{mmc}}(s) \cdot\left(\boldsymbol{T}_{a b c / \alpha \beta \pm}^{\mathrm{hss}}\right)^{-1}
$$

where $\boldsymbol{T}_{a b c / \alpha \beta \pm}^{\mathrm{hss}}=\operatorname{diag}\left(\ldots, \boldsymbol{T}_{a b c / \alpha \beta \pm}, \boldsymbol{T}_{a b c / \alpha \beta \pm}, \boldsymbol{T}_{a b c / \alpha \beta \pm}, \ldots\right)$.

\section{Asymmetric Effects on the MIMO Impedance}

The accuracy of the established $Z_{\alpha \beta \pm}^{\mathrm{mmc}}(s)$ will be validated by comparing with the measured frequency responses (electrical and control parameters of the MMC are given in Table I [7]). Due to space limitations, only the verification results of matrix elements from $1^{\text {st }}$ row to $7^{\text {th }}$ row and $1^{\text {st }}$ column to $7^{\text {th }}$ column are presented in Fig. 3, including symmetric conditions and asymmetric conditions. As can be seen from the comparative results, the calculated frequency responses are consistent with the frequency scanning results, confirming the accuracy of the established MIMO impedance model.

For symmetric conditions, it can be seen from Fig. 3(a) that there only exist the elements denoted by black squares (diagonal components) and red squares (mirror frequency coupling [19]), while the values of remaining elements are nearly zero (sporadic red points, e.g., in $1^{\text {st }}$ row and $3^{\text {rd }}$ column, are caused by measurement singularities, which will not influence the correctness of the analytical model). It has been proven in Refs [16], [25] that the red squares separated by $2 \omega_{1}$ can be obtained using the frequency shifting properties, and thus a $2 \times 2$ impedance matrix can contain all information of the $14 \times 14$ MIMO impedance. This again demonstrates the feasibility of the $2 \times 2$ impedance model developed for symmetric MMC analysis.

For asymmetric conditions, it can be seen from Fig. 3(b) that more coupling terms will appear such as the elements denoted

TABLE I

PARAMETERS OF THE MMC

\begin{tabular}{llll}
\hline \hline Items & Value & Items & Value \\
\hline Rated power & $50 \mathrm{MW}$ & PQ outer loop $k_{\mathrm{p}}^{\mathrm{PQ}}$ & $2 \mathrm{e}-6$ \\
Nominal Frequency & $50 \mathrm{~Hz}$ & PQ outer loop $k_{\mathrm{i}}^{\mathrm{PQ}}$ & $2 \mathrm{e}-5$ \\
Arm inductance & $0.36 \mathrm{H}$ & Current loop $k_{\mathrm{pi}}$ & 15 \\
SM capacitance & $1.4 \mathrm{mF}$ & Current loop $k_{\mathrm{ii}}$ & 3000 \\
Rated ac voltage & $166 \mathrm{kV}$ & PLL $k_{\mathrm{p}}^{\mathrm{pll}}$ & 50 \\
Arm resistance & $0.2 \Omega$ & PLL $k_{\mathrm{i}}^{\mathrm{pll}}$ & 900 \\
SM number per arm & 200 & CCSC $k_{\mathrm{pc}}$ & 10 \\
DC link voltage & $320 \mathrm{kV}$ & CCSC $k_{\mathrm{ic}}$ & 2000 \\
\hline \hline
\end{tabular}




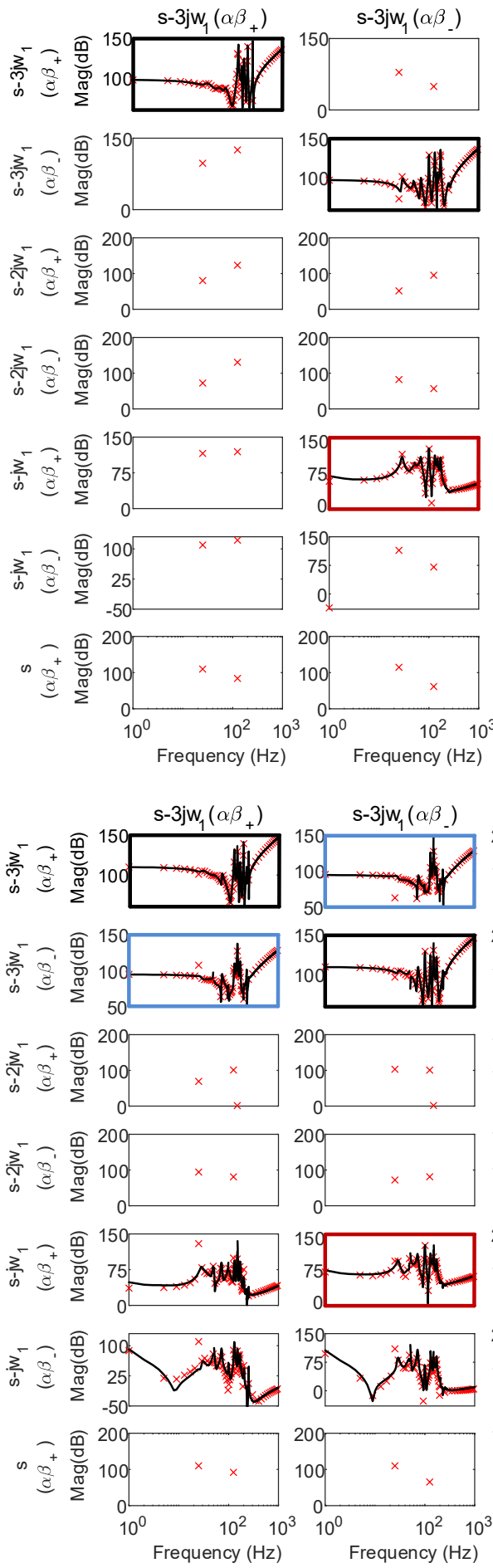

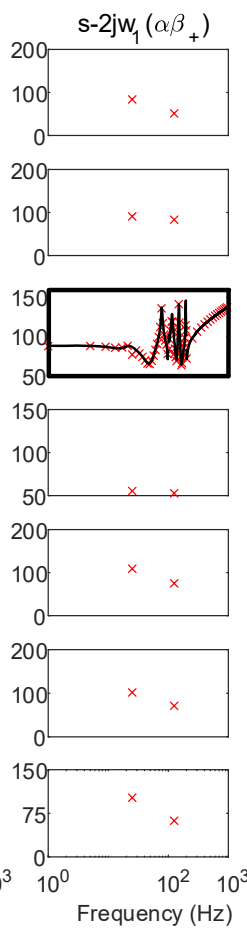
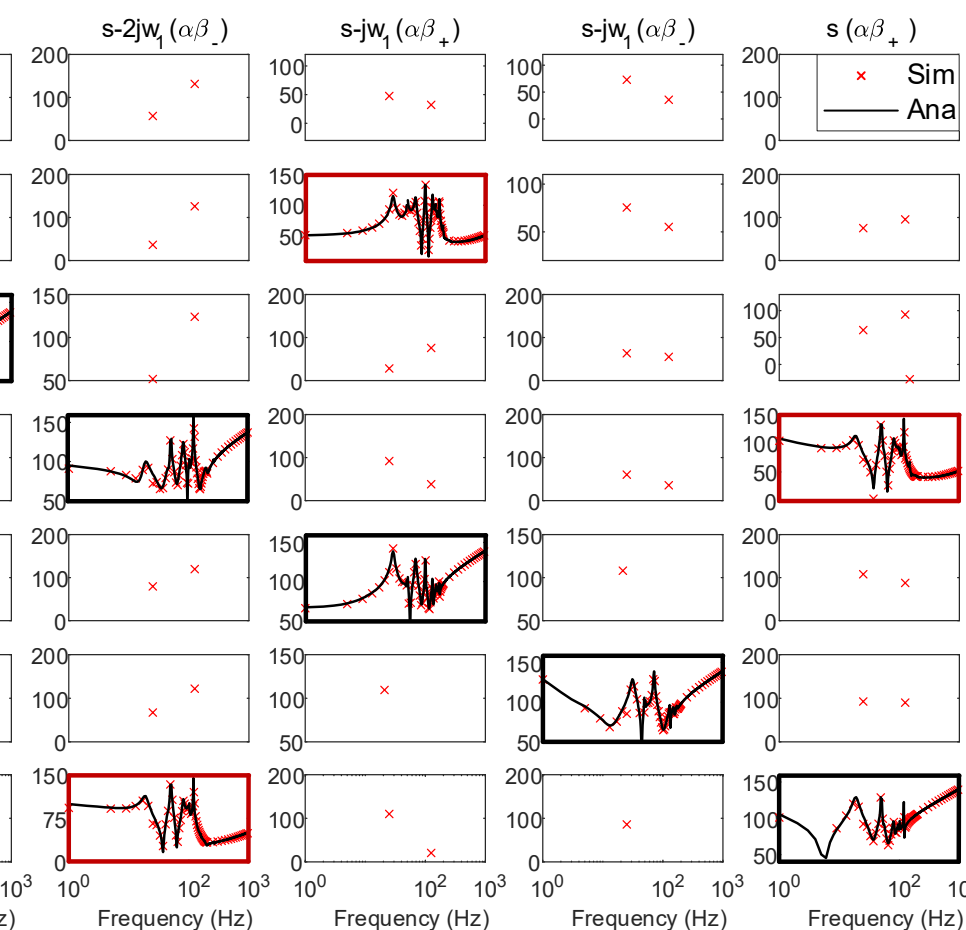

(a)
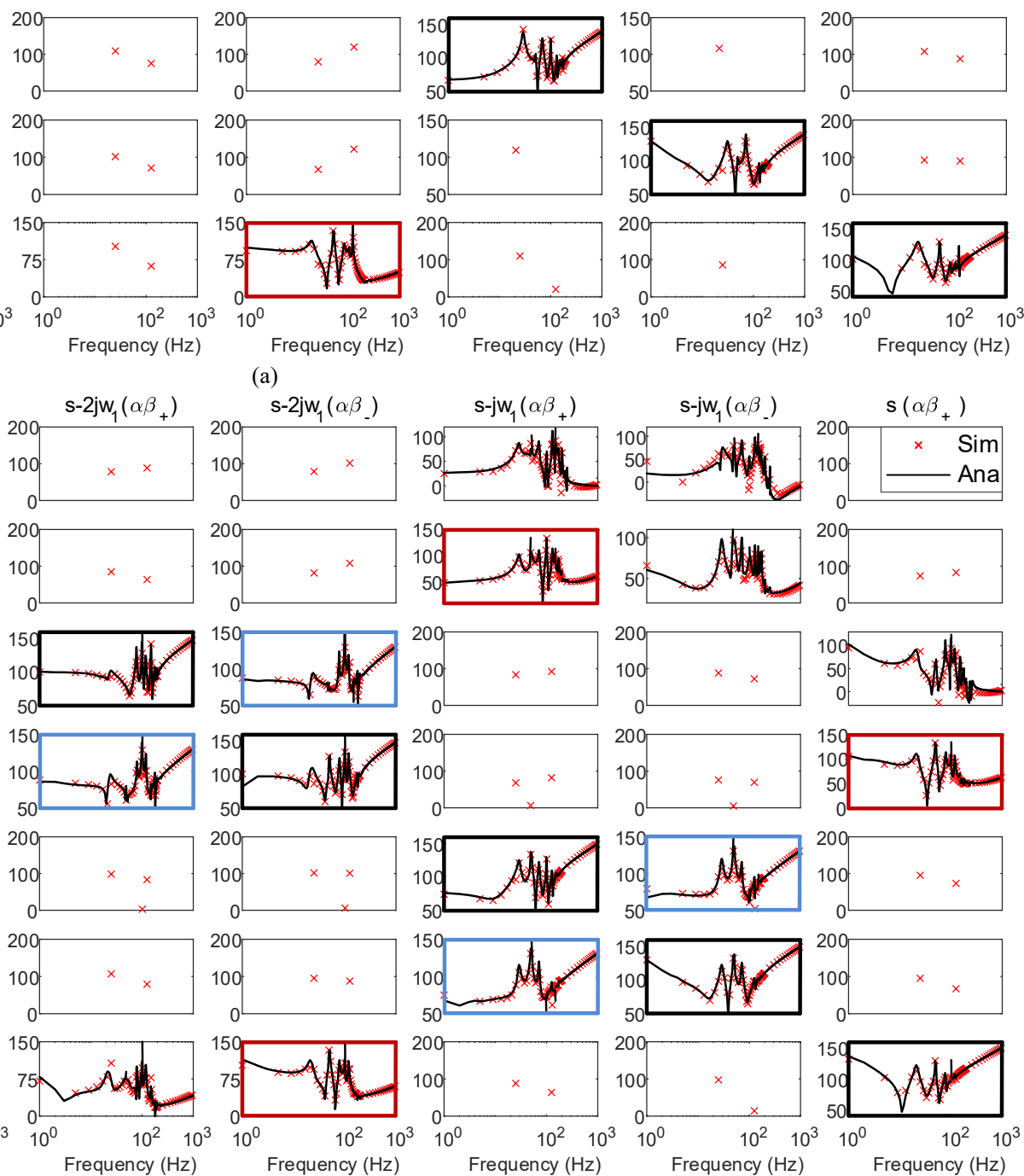

(b)
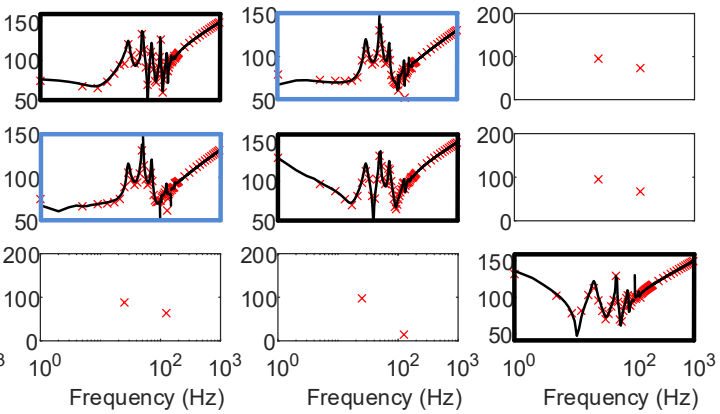

Fig. 3. Impedance verification of the $Z_{\alpha \beta \pm}^{\mathrm{mmc}}(s)[1: 7,1: 7]$, (a) symmetric condition; (b) asymmetric branch condition $\left(L_{\mathrm{arm} a}=3 \times 0.36 \mathrm{H}\right)$.

by blue squares (sequence coupling). A detailed introduction of various couplings mentioned above, including mirror frequency coupling and sequence coupling, is given in Appendix B. It becomes arguable whether the $2 \times 2$ impedance matrix as truncated in the way of the symmetric cases is still accurate enough to represent the whole MIMO impedance under asymmetric conditions. Therefore, it is desirable to establish a model reduction criterion, which will be presented in the forthcoming section.

\section{Block Diagonal Dominance-Based Reduced- ORDER IMPEDANCE MODEL}

The above discussion reveals that the main reason for the increasing in the dimension of the MMC impedance is the appearance of multiple non-diagonal elements. Qualitatively, if non-diagonal elements are 'weak' enough compared to diagonal ones, a simpler model can be obtained by retaining only diagonal elements. Such understanding can be rephrased 
as the partial interaction or the diagonal dominance, proposed by Rosenbrock [21] seeking to reduce a multivariable problem to one amenable to classical techniques. Then, such diagonal dominance concept was promoted to the block matrix and the block diagonal dominance (BDD) theory [22], [23] was developed. Based on such BDD concept, in this section, a model reduction criterion for the MIMO impedance of the $\mathrm{MMC}$ is developed considering various asymmetric scenarios.

\section{A. BDD-based Simplified Stability Criterion}

The BDD theory was originally applied in simplifying the design of the MIMO control system [21], while the same notion can be deployed for model reduction. The essence of the model reduction criterion can be summarized as: 'if the MIMO system belongs to $B D D$, the stability conclusion can be accurately predicted by only using block diagonal components instead of the whole MIMO matrix'. Of primary importance in this simplified stability criterion is the definition of a BDD system, which will be introduced at first. Then, the BDD-based stability criterion will be brought in detail.

\section{1) Definition of the BDD System}

The block diagonally dominant matrix is the generalized form of the diagonally dominant matrix ${ }^{1}$. Let $\boldsymbol{Z}(s)$ be a $n \times n$ rational matrix, partitioned in the following manner:

$$
\boldsymbol{Z}(s)=\left[\begin{array}{cccc}
\boldsymbol{z}_{1,1}(s) & \boldsymbol{z}_{1,2}(s) & \cdots & \boldsymbol{z}_{1, N}(s) \\
\boldsymbol{z}_{2,1}(s) & \boldsymbol{z}_{2,2}(s) & \cdots & \boldsymbol{z}_{2, N}(s) \\
\vdots & & & \vdots \\
\boldsymbol{z}_{N, 1}(s) & \boldsymbol{z}_{N, 2}(s) & \cdots & \boldsymbol{z}_{N, N}(s)
\end{array}\right]
$$

where the submatrices $\boldsymbol{z}_{i i}(s)$ are square of order $n_{i i}, 1 \leq i \leq N$.

Definition 1: Let D be a closed elementary contour in C, i.e., complex plane. Suppose $\boldsymbol{z}_{i i}(s)$ has no pole and is nonsingular on $D(i=1, \cdots, N)$, the matrix $\boldsymbol{Z}(s)$ is block diagonally dominant on the contour $D$ if for each son $D$ :

$$
\left\|z_{i i}(s)\right\|_{2} \geq \sum_{j=1, j \neq i}^{N}\left\|z_{i j}(s)\right\|_{2}
$$

where the \|\|$_{2}$ denotes the 2-norm (Euclidean norm), i.e., the square root of maximum eigenvalue of $\boldsymbol{z}_{i i}^{*}(s) \boldsymbol{z}_{i i}(s)$.

Since $\boldsymbol{z}_{i i}(s)$ is nonsingular, (19) can be rewritten as:

$$
\underbrace{\frac{\sum_{j=1, j \neq i}^{N}\left\|\boldsymbol{z}_{i j}(s)\right\|_{2}}{\left\|\boldsymbol{z}_{i i}(s)\right\|_{2}}}_{k_{i}(s)} \leq 1
$$

where the ratio is defined as the $k_{i}(s)(i=1, \cdots, N)$ for convenience.

The Definition 1 means that if $k_{i}(s) \leq 1(i=1, \cdots, N)$ across the frequency range of interest (e.g. $1 \sim 1 \mathrm{kHz}$ ), the $\boldsymbol{Z}(s)$ can be regarded as a BDD matrix/system. Particularly, if the $Z(s)$ is a

\footnotetext{
${ }^{1}$ In mathematics, a square matrix is said to be diagonally dominant if, for every row of the matrix, the magnitude of the diagonal entry in a row is larger
}

block diagonal matrix, i.e., only principal block diagonal elements $\boldsymbol{z}_{i i}(s) \neq 0$, then $k_{i}(s)(i=1, \cdots, N)$ maintain 0 . To be more intuitive, the ratio of the number of frequency points whose $k_{i}(s)>1$ to the number of considered frequency points $\eta$ is defined as $\xi_{i}$ :

$$
\xi_{i}=\frac{\text { Number }\left(k_{i}\left(s_{f}\right)>1\right)}{\eta}, \quad 1 \leq f \leq \eta
$$

where if $\xi_{i} \approx 0$ for $i=1, \cdots, N\left(\right.$ i.e., $k_{i}(s) \leq 1$ for nearly all frequency points), the $\boldsymbol{Z}(s)$ can be regarded as a BDD system.

Compared with the diagonal dominance theory [21], the robustness of the Definition 1 is greatly improved due to its ability of partitioning the $\boldsymbol{Z}(s)$ with flexible dimensions. From the experience of symmetric cases [15]-[17], the dimension of the submatrix $\boldsymbol{z}_{i i}(s)$ can be set as $2\left(n_{i i}=2\right)$, the simplest form containing at least one pair of coupling terms. It is noted that if Definition 1 cannot be complied with the $2 \times 2$ dimension, then the $n_{i i}$ needs to be increased.

2) BDD-based Nyquist Stability Criterion

The equivalent circuit of the grid-tied MMC and its closedloop system are shown in Fig. 4, where $\boldsymbol{F}(s)$ represents the MMC admittance $\boldsymbol{Y}_{\text {mmc }}^{\text {hss }}(s)$ and $\boldsymbol{G}(s)$ represents the grid impedance $\boldsymbol{Z}_{\text {grid }}^{\mathrm{hss}}(s)$.

Lemma 1: Suppose $\boldsymbol{F}(s)$ has no RHP-pole, and $\left(\boldsymbol{F}(s)^{-1}+\right.$ $G(s))$ is block diagonally dominant on the contour D. Let $\operatorname{det}\left(\boldsymbol{F}_{i i}(s)^{-1}+\boldsymbol{G}_{i i}(s)\right)$ map $D$ into $\Gamma_{i}$ which encircles the origin $\Gamma_{i}$ times clockwise $(i=1, \cdots, N)$. Then, the closed-loop system is asymptotically stable if and only if

$$
\sum_{i=1}^{N} \Gamma_{i}=-p_{0}
$$

where $p_{0}$ represents the number of open-loop system RHPpoles. The rigorous proof for the Lemma 1 can be referred to Ref [22].

Since the studied closed-loop system is type of $\boldsymbol{Z}+\boldsymbol{Y}$ [26] system and each submodule is stable in stand-alone mode, there will be no RHP-poles in the sub-system $\boldsymbol{F}(s)$ and $\boldsymbol{G}(s)$, i.e., $p_{0}=0$. What's more, the $\boldsymbol{F}^{-1}(s)$ (i.e., the impedance of the MMC) generally doesn't surround the origin if with the proper design, which indicates that $\Gamma_{i}\left(\operatorname{det}\left(\boldsymbol{F}_{i i}{ }^{-1}\right)\right)=0$ and thus $\Gamma_{i}\left(\operatorname{det}\left(\boldsymbol{F}_{i i}{ }^{-1}+\boldsymbol{G}_{i i}\right)\right)=\Gamma_{i}\left(\operatorname{det}\left(\boldsymbol{I}_{i i}+\boldsymbol{G}_{i i} \boldsymbol{F}_{i i}\right)\right)$. Consequently, the stability of the closed-loop system can be evaluated equivalently using the impedance-ratio $\boldsymbol{G}_{i i}(s) \boldsymbol{F}_{i i}(s)$.

The Lemma 1 is able to reduce the stability analysis of a $N$ dimensional MIMO problems to that of $N$ separate 1-

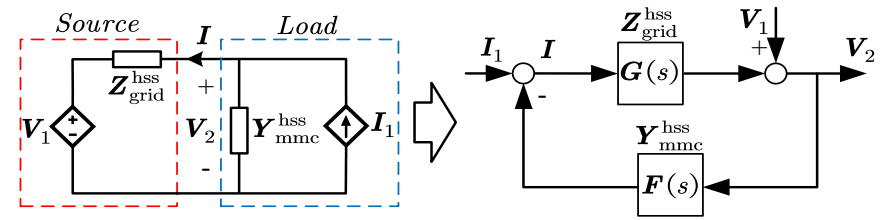

Fig. 4. Equivalent circuit and closed-loop system

than or equal to the sum of the magnitudes of all the other (non-diagonal) entries in that row. 
dimensional problems. The stability judgement only needs to inspect the encirclement situation of $(-1, j 0)$ using $N$ separate $\boldsymbol{G}_{i i}(s) \boldsymbol{F}_{i i}(s)$ (also known as the impedance ratio). What's more, the HSS model is possessed of frequency shifting properties [25], e.g., $\boldsymbol{G}_{11}(s) \boldsymbol{F}_{11}(s)=\boldsymbol{G}_{44}\left(s-4 j \omega_{1}\right) \boldsymbol{F}_{44}\left(s-4 j \omega_{1}\right)$. Aided with this property, the stability analysis can be further simplified by using one single block diagonal submatrix, which contains all information of other block diagonal submatrices.

In conclusion, if the Lemma 1 was satisfied, the highdimensional MIMO impedance can be reduced as a $2 \times 2$ matrix. The stability conclusion can be accurately predicted by using one $2 \times 2$ impedance ratio $\boldsymbol{G}_{i i}(s) \boldsymbol{F}_{i i}(s)$, e.g., $\boldsymbol{G}_{44}(s) \boldsymbol{F}_{44}(s)$. Compared with the Gershgorin circle theory [27], [28], the conservativeness of the Lemma 1 has been reduced a lot, the reason behind which can be referred to Ref [22].

\section{B. BDD System Characteristics Analysis}

A useful model reduction criterion is obtained from the above analysis, while its feasibility depends on whether the requirements of the Lemma $\mathbf{1}$ can be satisfied, i.e., the studied MIMO system belongs to BDD. Around this, the BDD characteristics analysis of the established MIMO impedance is carried out considering various asymmetry degrees (weak, medium and strong). The mentioned three kinds of asymmetry degrees are defined based on the results of the rigorous BDD inspection.

\section{1) $B D D$ Characteristics in $\alpha-\beta$ Domain}

At first, the BDD characteristics of the derived MIMO impedance is analyzed in $\alpha-\beta$ domain. The grid impedance $Z_{\alpha \beta \pm}^{\text {grid }}(s)$ in $\alpha$ - $\beta$ domain can be transformed from the three-phase grid impedance $\boldsymbol{Z}_{x}^{\text {grid }}(s)$ (e.g., $\left.\boldsymbol{Z}_{a}^{\text {grid }}(s)=R_{\text {ga }}+s L_{\text {ga }}\right)$ using (17). To be more intuitive, based on (20) and (21), the $k_{4}(s)$ and $\xi_{4}$ corresponding to the ' $s$ ' is calculated as an example.

$$
\left\{\begin{array}{l}
k_{4}(s)=\frac{\sum_{j=1, j \neq 4}^{7}\left\|\boldsymbol{F}_{4 j}(s)^{-1}+\boldsymbol{G}_{4 j}(s)\right\|_{2}}{\left\|\boldsymbol{F}_{44}(s)^{-1}+\boldsymbol{G}_{44}(s)\right\|_{2}} \\
\xi_{4}=\frac{\text { Number }\left(k_{4}\left(s_{f}\right)>1\right)}{\eta}
\end{array}\right.
$$

where the considered $\eta=1 \mathrm{kHz}$ (i.e., considering one thousand frequency points).

Qualitatively, the system asymmetry will be deepened by increasing the single-phase (e.g., A phase) branch inductance or single-phase grid impedance. The BDD characteristics of the grid-tied MMC with unbalanced A-phase inductance is presented in Fig. 5(a) (red curve denotes the $\alpha-\beta$ domain), where the $\xi_{4}$ decreases as the $L_{\operatorname{arm} a}$ increases and approaches to 0 (BDD) when $L_{\mathrm{arm} a}>3.7 \times 0.36 \mathrm{H}\left(L_{\mathrm{arm} b}=L_{\mathrm{arm} c}=0.36 \mathrm{H}\right)$. Four examples of $k_{4}(s)$ are given in Fig. 5(a), from which it can be observed obviously that the number of frequency points whose $k_{4}(s)>1$ gradually decreases. Similarly, the BDD characteristics of grid-tied MMC with unbalanced A-phase grid impedance is presented in Fig. 5(b) (red curve denotes $\alpha-\beta$ domain). When the $X_{\mathrm{g} a}$ is larger than $10.5 \times 47.3 \Omega\left(X_{\mathrm{g} b}=X_{\mathrm{g} c}=47.3 \Omega\right)$, the BDD can be met in $\alpha-\beta$ domain. Based on these two cases, it can be concluded that when the asymmetry degree of the main circuit is deepened to some extent, the MIMO impedance at ' $s$ ' become a BDD system by using the impedance model in $\alpha-\beta$ domain.

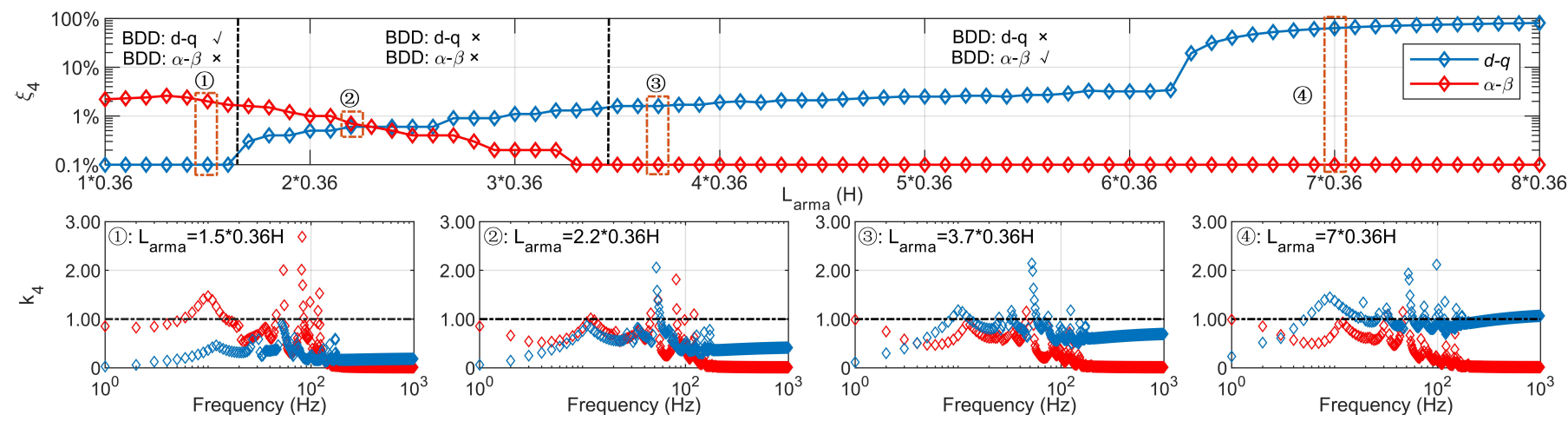

(a)
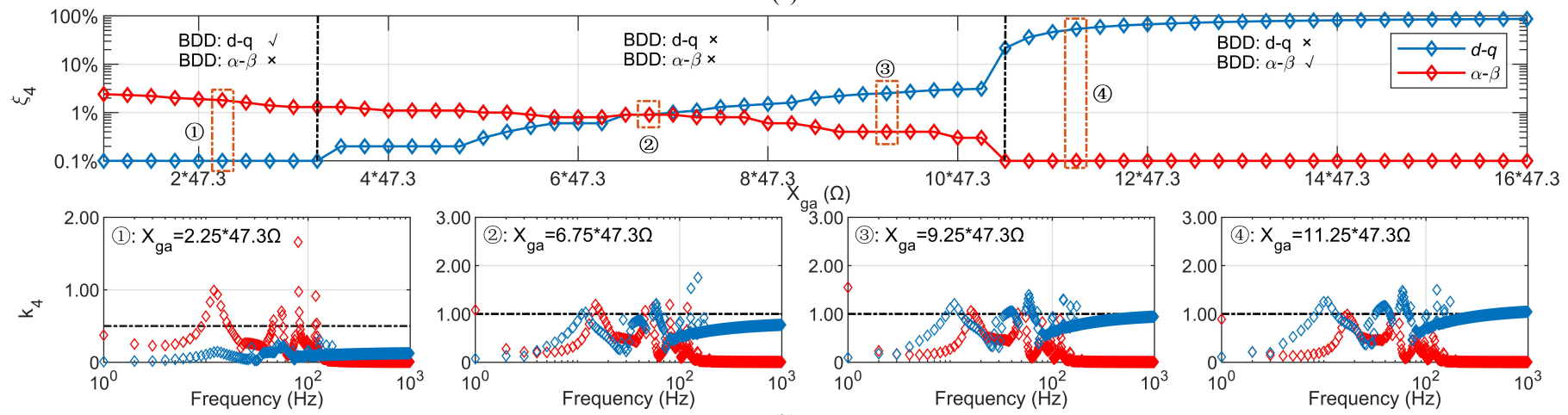

(b)

Fig. 5. BDD characteristics of the MIMO impedance matrix in $\alpha-\beta$ and $d-q$ domain, (a) Asymmetric branch conditions; (b) Asymmetric ac-grid conditions 


\section{2) BDD characteristics in $d-q$ domain}

From the Fig. 5, it should be noted that there still exists an area (e.g., $L_{\mathrm{arm} a}<3.7 \times 0.36 \mathrm{H}$ or $X_{\mathrm{g} a}<10.5 \times 47.3 \Omega$ ), where the BDD cannot be acquired. To improve the applicability of the model reduction criterion, a permutation matrix is commonly applied to rearrange the components' distribution of the MIMO matrix without affecting its eigenvalues, so that the new MIMO matrix may become BDD. This tech was frequently used in the design of MIMO control system and a suitable permutation matrix is usually acquired via the graph theory [21]. In this paper, as will be demonstrated, the rotation matrix of the $\alpha-\beta$ domain to the $d-q$ domain [18] is indeed a good permutation matrix [25] with sound physical implications, the effect of which can be interpreted as selecting the appropriate reference frame for modelling and analysis. The rotation matrix at $h \omega_{1}$ harmonic can be expressed as:

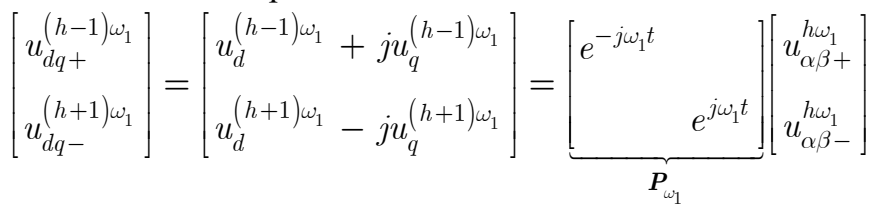

By extending (24) in the harmonic domain, the MIMO impedance $\boldsymbol{Z}_{\alpha \beta \pm}^{\text {grid }}(s)$ can be transformed into the $\boldsymbol{Z}_{d q \pm}^{\text {grid }}(s)$ as:

$$
\boldsymbol{Z}_{d q \pm}^{\mathrm{hss}}=\boldsymbol{P}_{\omega_{1}}^{\mathrm{hss}} \cdot \boldsymbol{Z}_{\alpha \beta \pm}^{\mathrm{hss}} \cdot\left(\boldsymbol{P}_{\omega_{1}}^{\mathrm{hss}}\right)^{-1}
$$

where the permutation matrix $\boldsymbol{P}_{\omega_{1}}^{\mathrm{hss}}$ is the Toeplitz form of the $\boldsymbol{P}_{\omega_{1}}$ as:

$$
\boldsymbol{P}_{\omega_{1}}^{\mathrm{hss}}=\left[\begin{array}{llllllll} 
& & \ddots & & & & & \\
& & & 1 & 0 & & & \\
\ddots & & & 0 & 0 & & & \\
& 0 & 0 & & & 1 & 0 & \\
& 0 & 1 & & & 0 & 0 & \\
& & & 0 & 0 & & & \ddots \\
& & 0 & 1 & & & \\
& & & & \ddots & &
\end{array}\right]
$$

The effect of the permutation matrix is studied in Fig. 6, where the lighter the square color, the weaker the coupling strength in accordance with the results of Fig. 3. For symmetric cases, there will only exist elements denoted by black and red squares. It can be observed that the red squares in $\boldsymbol{Z}_{\alpha \beta \pm}^{\mathrm{mmc}}(s)$ are moved to the principal block diagonal in $\boldsymbol{Z}_{d q \pm}^{\mathrm{mmc}}(\mathrm{s})$, while the blue squares are moved out. In fact, the coupling terms denoted by blue squares are the so-called sequence coupling, whose strength is closely associated with the three-phase asymmetry. The coupling terms denoted by the red squares are the so-called mirror frequency coupling (MFC) [18], whose strength is mainly affected by the control asymmetry (e.g., $d-q$ asymmetry [29]).

Now, the BDD characteristics of the grid-tied MMC will be

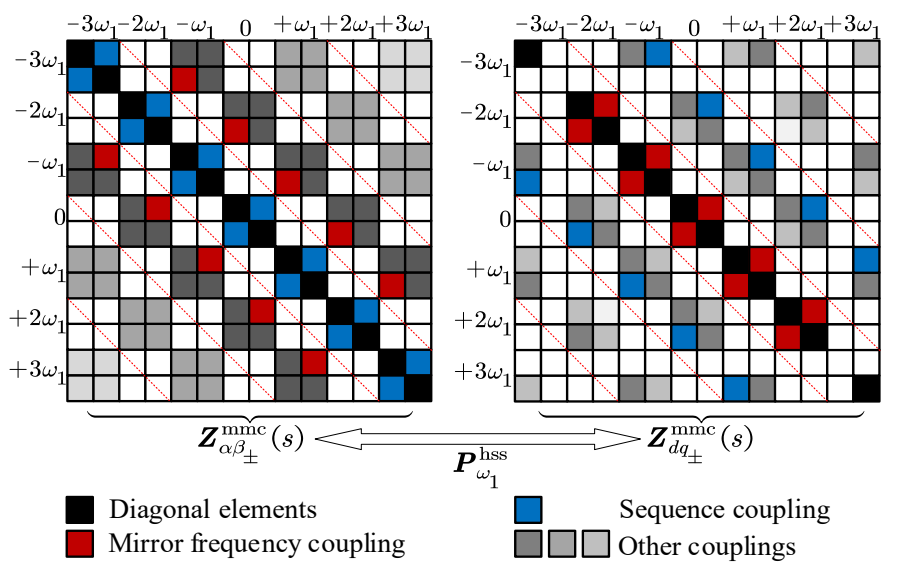

Fig. 6. The effect of the permutation matrix $\boldsymbol{P}_{\omega_{1}}^{\mathrm{hss}}$ on the impedance distribution

analyzed in $d-q$ domain and the Fig. 5 can be used again for illustration. As shown in the blue curve in Fig. 5(a), $\xi_{4}$ in $d-q$ domain increases as the $L_{\operatorname{arm} a}$ increases and approaches to 0 (BDD) when $L_{\mathrm{arm} a}<1.7 \times 0.36 \mathrm{H}$. Similarly, as shown in the blue curve in Fig. 5(b), $\xi_{4}$ in $d-q$ domain increases as the $X_{\mathrm{g} a}$ increases and approaches to 0 (BDD) when $X_{\mathrm{g} a}<3.25 \times 47.3 \Omega$. Based on these two cases, it can be concluded that when the asymmetry degree of the main circuit is weak, the MIMO impedance at ' $s$ ' can become a BDD system by using the impedance model in $d-q$ domain.

\section{BDD-based Reduced-order Model}

Based on the qualitative analysis presented above, the criterion for different asymmetry degrees and the corresponding reduced-order model can be given as:

Strong Asymmetry: If each $\boldsymbol{\xi}_{i}$ of the $\left(\boldsymbol{F}(s)^{-1}+\boldsymbol{G}(s)\right)$ is close to 0 in $\alpha-\beta$ domain, it equivalently means that the overall system satisfies the prerequisite of Lemma 1 in $\alpha-\beta$ domain. This evidence further implies that three-phase asymmetric effect (i.e., sequence coupling) dominates the small-signal characteristics of the models. Therefore, a system follows such a condition is defined as the 'strong asymmetry'. Under such circumstance, the $2 \times 2$ reduced-order model in $\alpha$ - $\beta$ domain can be applied.

Weak Asymmetry: If each $\boldsymbol{\xi}_{i}$ of the $\left(\boldsymbol{F}(s)^{-1}+\boldsymbol{G}(s)\right)$ is close to 0 in $d-q$ domain, it equivalently means that the overall system satisfies the prerequisite of Lemma $\mathbf{1}$ in $d-q$ domain. This evidence further implies that control asymmetric effect (i.e., mirror frequency coupling) dominates the small-signal characteristics of the models. Therefore, a system follows such a condition is defined as the 'weak asymmetry'. Under such circumstance, the $2 \times 2$ reduced-order model in $d-q$ domain can be applied.

Medium Asymmetry: If some $\boldsymbol{\xi}_{i}$ of the $\left(\boldsymbol{F}(s)^{-1}+\boldsymbol{G}(s)\right)$ are larger than 0 both in $d-q$ and $\alpha-\beta$ domain, it equivalently means that the overall system can satisfy the prerequisite of Lemma 1 neither in $d-q$ domain nor $\alpha-\beta$ domain. This evidence further implies that neither control asymmetry nor three-phase asymmetry can be ignored. Therefore, a system follows such a condition is defined the 'medium asymmetry'. The proposed 
TABLE II

IMPEDANCE REDUCTION

\begin{tabular}{cccc}
\hline \hline $\begin{array}{c}d-q \text { domain } \\
(i=1, \cdots, N)\end{array}$ & $\begin{array}{c}\alpha-\beta \text { domain } \\
(i=1, \cdots, N)\end{array}$ & $\begin{array}{c}\text { Asymmetry } \\
\text { Degree }\end{array}$ & Reduced model \\
\hline$\xi_{i} \approx 0$ & $\xi_{i}>0$ & Weak & $2 \times 2 d \pm j q$ \\
\hline$\xi_{i}>0$ & $\xi_{i} \approx 0$ & Strong & $2 \times 2 \alpha \pm j \beta$ \\
\hline$\xi_{i}>0$ & $\xi_{i}>0$ & Medium & $\backslash$ \\
\hline
\end{tabular}

reduced-order models either in $d-q$ or $\alpha$ - $\beta$ domain are invalid.

To be more intuitive, the above analysis is summarized in Table II, the idea of which is consistent with the qualitative understanding of the MMC frequency response: (1) when the three-phase system is symmetric or weak asymmetric, the frequency response will be dominated by the MMC control, which is mostly based on the $d q$-frame, thus it is favorable to analyze the system at $d q$-frame to achieve a compact block matrix; (2) in contrast, if the system is more towards the threephase asymmetric (strong asymmetric), then the effect of threephase asymmetry may take over that of the control, resulting in that the $\alpha \beta$-impedance will be more compact than the $d q$ one. However, an exact conclusion of whether the studied grid-tied MMC is a BDD system can only be drawn by evaluating each $\xi_{i}$ of the $\left(\boldsymbol{F}(s)^{-1}+\boldsymbol{G}(s)\right)$ using the (20) and (21).

\section{CAse Studies}

In this section, the effectiveness of the proposed reducedorder model is tested by using two common asymmetric scenarios, i.e., unbalanced branch conditions and unbalanced ac-grid conditions.

\section{A. Case Description}

The asymmetric parameters of the adopted six cases are listed in Table III, while other parameters remain symmetric as given in Table I. The marginally stable/unstable states (i.e., the critical condition) are realized by adjusting the internal resistance of the ac-grid.

According to the Section III.A, the stability of the asymmetric grid-tied MMC system can be determined by using any $2 \times 2$ impedance ratio $\boldsymbol{G}_{i i}(s) \boldsymbol{F}_{i i}(s)$, as long as the prerequisites of the Lemma 1 are satisfied. Here, the impedance ratio corresponding to ' $s$ ' $(i=4)$ is chosen which can be rewritten in $\alpha-\beta$ domain and $d-q$ domain as:

$$
\begin{aligned}
& \boldsymbol{L}_{\alpha \beta \pm}=\left\{\boldsymbol{Z}_{\alpha \beta \pm}^{\text {grid }}\right\}_{44}(s) \cdot\left\{\boldsymbol{Y}_{\alpha \beta \pm}^{\mathrm{mmc}}\right\}_{44}(s) \\
& \boldsymbol{L}_{d q \pm}=\left\{\boldsymbol{Z}_{d q \pm}^{\text {grid }}\right\}_{44}(s) \cdot\left\{\boldsymbol{Y}_{d q \pm}^{\mathrm{mmc}}\right\}_{44}(s)
\end{aligned}
$$

where both the $14 \times 14 \boldsymbol{Z}_{\alpha \beta / d q \pm}^{\text {grid }}(s)$ and $\boldsymbol{Y}_{\alpha \beta \pm / d q \pm}^{\mathrm{mmc}}(s)$ have been partitioned into the $7 \times 7$ block matrices.
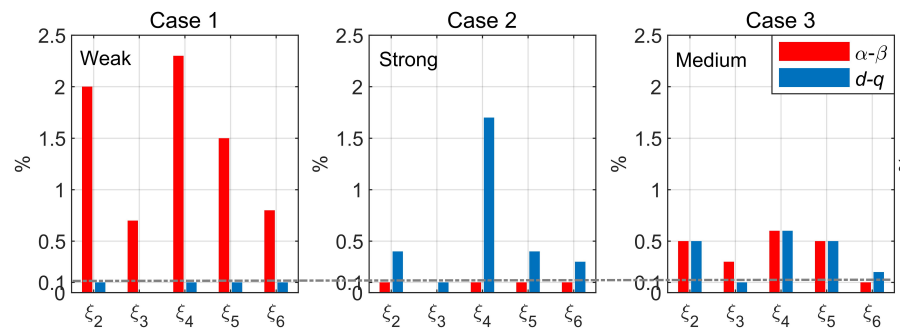

TABLE III

\begin{tabular}{|c|c|c|c|}
\hline & No. & MMC Parameters & Grid Parameters \\
\hline \multirow{3}{*}{$\begin{array}{l}\text { Asym } \\
\text { metric } \\
\text { MMC }\end{array}$} & $\# 1$ & $\boldsymbol{L}_{\mathrm{arm} b c}=0.36 \mathrm{H}, L_{\mathrm{arm} a}=1.5 L_{\mathrm{arm} b}$ & $X_{\mathrm{g} x}=37.7 \Omega$ \\
\hline & $\# 2$ & $\boldsymbol{L}_{\mathrm{arm} b c}=0.36 \mathrm{H}, L_{\mathrm{arm} a}=3.7 L_{\mathrm{arm} b}$ & $\boldsymbol{X}_{\mathrm{g} x}=40.8 \Omega$ \\
\hline & $\# 3$ & $\boldsymbol{L}_{\mathrm{arm} b c}=0.36 \mathrm{H}, L_{\mathrm{arm} a}=2.2 L_{\mathrm{arm} b}$ & $\boldsymbol{X}_{\mathrm{g} x}=39.3 \Omega$ \\
\hline \multirow{3}{*}{$\begin{array}{c}\text { Asym } \\
\text { metric } \\
\text { Grid }\end{array}$} & $\# 4$ & $\boldsymbol{L}_{\text {arm } x}=0.36 \mathrm{H}$ & $X_{\mathrm{g} a}=2.25 X_{\mathrm{g} b}, X_{\mathrm{g} b c}=28.1 \Omega$ \\
\hline & $\# 5$ & $\boldsymbol{L}_{\text {armx }}=0.36 \mathrm{H}$ & $X_{\mathrm{g} a}=11.25 X_{\mathrm{g} b}, X_{\mathrm{g} b c}=15.5 \Omega$ \\
\hline & $\# 6$ & $\boldsymbol{L}_{\mathrm{armx}}=0.36 \mathrm{H}$ & $X_{\mathrm{g} a}=6.75 X_{\mathrm{g} b}, X_{\mathrm{g} b c}=17.1 \Omega$ \\
\hline
\end{tabular}

CASE PARAMETERS

\section{B. Evaluation of Asymmetry Degrees}

Before carrying out the asymmetric stability analysis, the first step is to evaluate each $\boldsymbol{\xi}_{i}$ of the $\left(\boldsymbol{F}(s)^{-1}+\boldsymbol{G}(s)\right)$ so as to determine the asymmetry degree (weak/medium/strong) of the studied case. Since the MIMO matrix in $d-q$ domain is truncated at ' $\pm 3 \omega_{1}$ ' ( $i=1$ or 7$)$ referring to the Fig. 6 , only $\xi_{i}(i=2 \sim 6)$ need to be assessed.

The asymmetry degree evaluation of the six cases are presented in Fig. 7, from which it can be obtained that: (1) for the Case 1 , each $\xi_{i}(i=2 \sim 6)$ is close to 0 in $d-q$ domain which means the asymmetry degree of this case is weak and the $\boldsymbol{L}_{d q \pm}$ should be adopted; (2) for the Case 2, each $\xi_{i}(i=2 \sim 6)$ are close to 0 in $\alpha-\beta$ domain which means the asymmetry of this case is strong and the $\boldsymbol{L}_{\alpha \beta \pm}$ should be adopted; (3) for the Case 3, the $\xi_{i}$ is larger than 0 both in $d-q$ and $\alpha-\beta$ domain which means the asymmetry of this case is medium and the proposed reducedorder models are invalid. Similar conclusions can be obtained for the Case 4 6 that the $\boldsymbol{L}_{d q \pm}$ should be adopted for the Case 4, while the $\boldsymbol{L}_{\alpha \beta \pm}$ should be adopted for the Case 5. As for the Case 6, the proposed reduced-order models are invalid either in $d-q$ or $\alpha-\beta$ domain.

\section{Asymmetric Stability Analysis}

Based on the results of asymmetry degree assessment, the corresponding stability analysis is carried out to verify the accuracy of the proposed reduced-order models on marginally stable/unstable states predictions.

\section{1) Asymmetric branch conditions}

Case 1: As shown in Fig. 8(a), the $\boldsymbol{L}_{d q \pm}$ can predict the correct stability conclusion, i.e., the marginally stable state with $\boldsymbol{R}_{\mathrm{g} x}=8 \Omega$ and the marginally unstable state with $\boldsymbol{R}_{\mathrm{g} x}=6 \Omega$, which conforms with the time-domain simulations. A counterexample using $\boldsymbol{L}_{\alpha \beta \pm}$ is also given in Fig. 8(a), where an optimistic stability conclusion is obtained, i.e., the stable state with $\boldsymbol{R}_{\mathrm{g} x}=8 \Omega$ and $6 \Omega$. Now, it has been validated that the
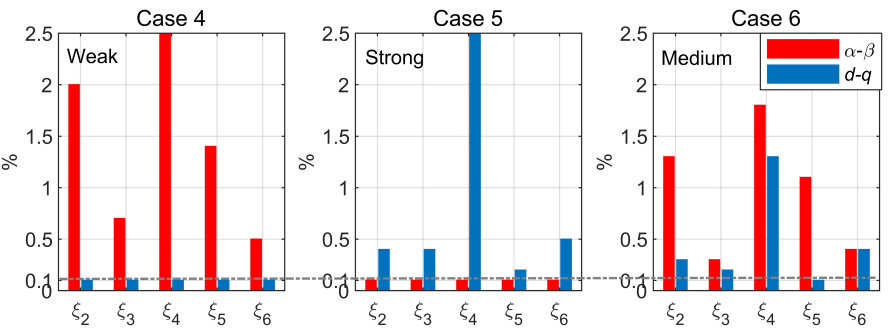

Fig. 7. Asymmetry degree evaluation. 

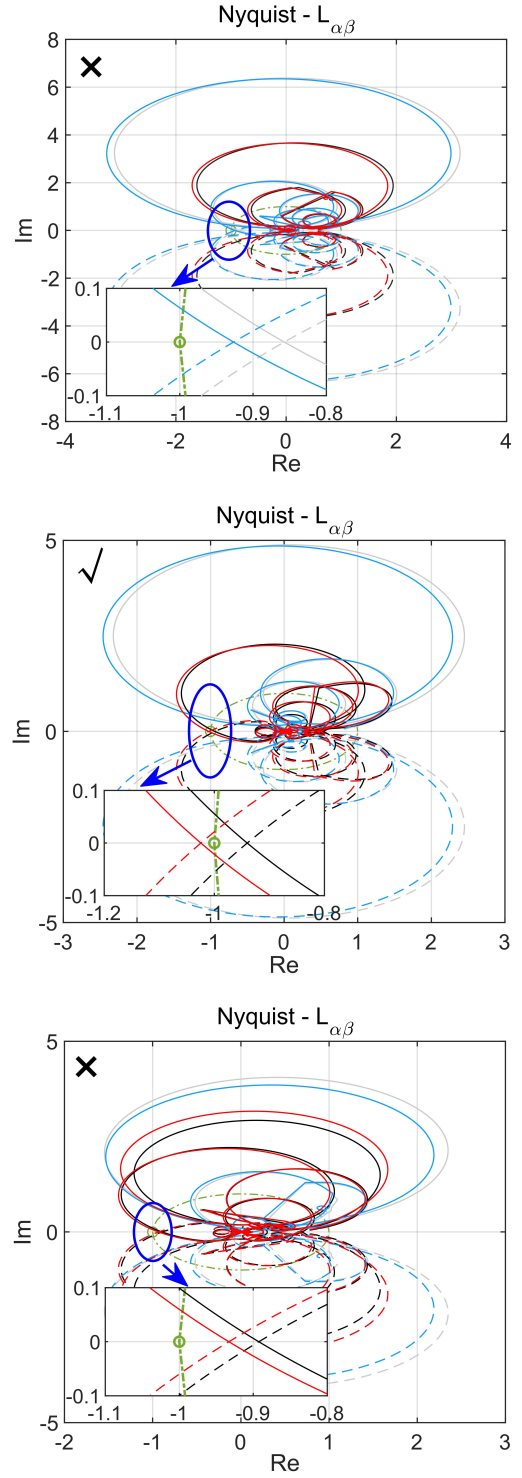

Previous State

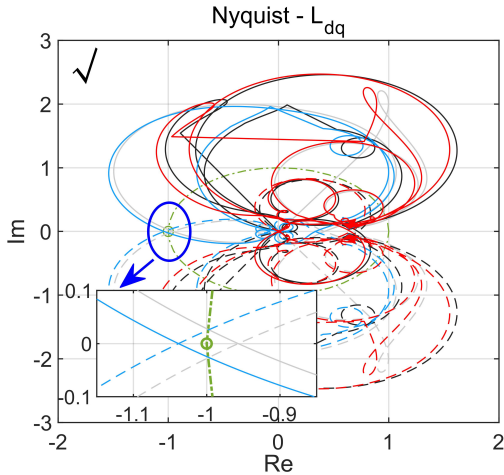

(a)

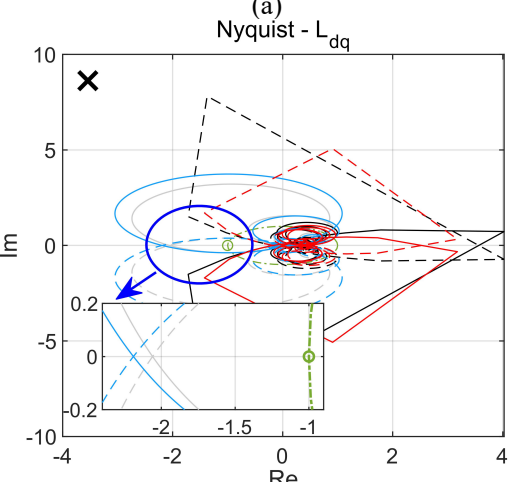

(b)

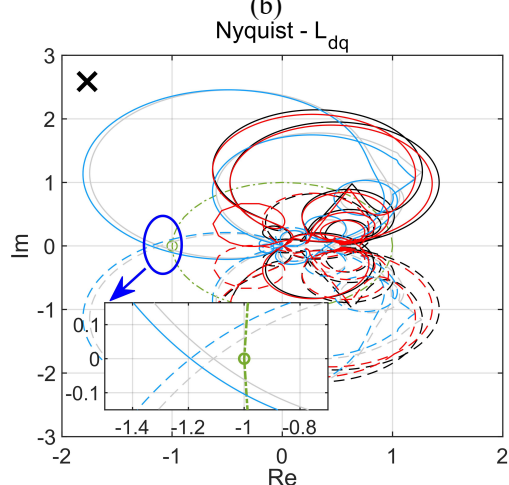

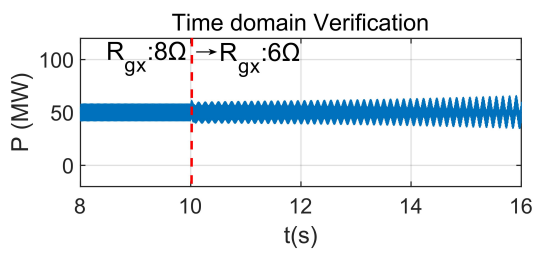
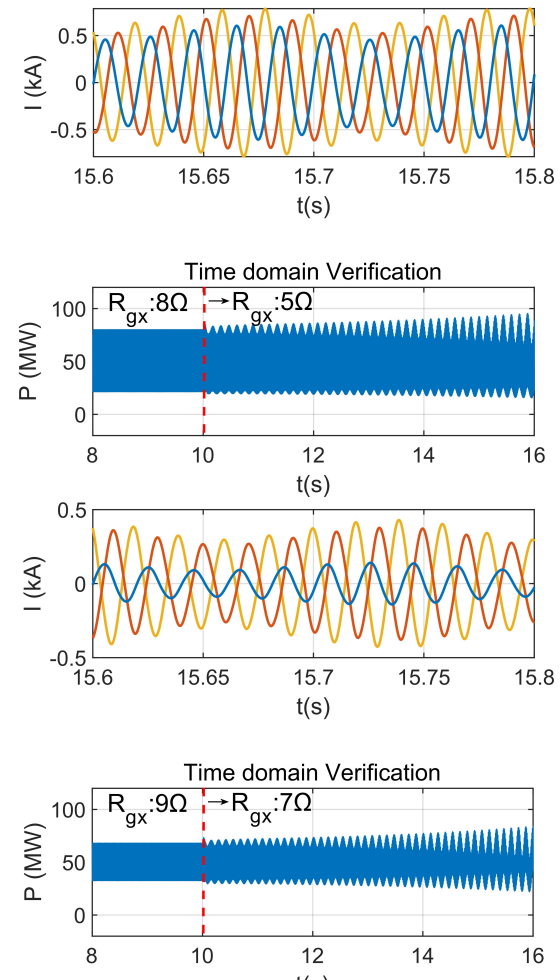

$t(s)$

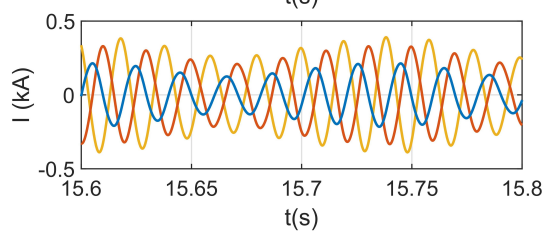

(c) Latter State

Fig. 8. Theoretical stability analysis and time-domain verifications, (a) Case 1; (b) Case 2; (c) Case 3.

accurate stability conclusion can be obtained by using the $2 \times 2$ impedance in $d-q$ domain under the Weak Asymmetry.

Case 2: As shown in Fig. 8(b), the $\boldsymbol{L}_{\alpha \beta \pm}$ can predict the correct stability conclusion, i.e., the marginally stable state with $\boldsymbol{R}_{\mathrm{g} x}=8 \Omega$ and the marginally unstable state with $\boldsymbol{R}_{\mathrm{g} x}=5 \Omega$, which conforms with the time-domain simulations. A counterexample using $\boldsymbol{L}_{d q \pm}$ is also given in Fig. 8(b), where an extremely pessimistic conclusion is obtained, i.e., the unstable state with $\boldsymbol{R}_{\mathrm{g} x}=8 \Omega$ and $5 \Omega$. Now, it has been validated that the accurate stability conclusion can be obtained by using the $2 \times 2$ impedance in $\alpha-\beta$ domain under the Strong Asymmetry.

Case 3: As shown in Fig. 8(c), compared with the timedomain results, neither the $\boldsymbol{L}_{\alpha \beta \pm}$ nor the $\boldsymbol{L}_{d q \pm}$ can give an accurate stability conclusion. The obtained stability result is more pessimistic using $\boldsymbol{L}_{d q \pm}$, while more optimistic using $\boldsymbol{L}_{\alpha \beta \pm}$.

In practical systems, there basically will only exist Weak
Asymmetry under asymmetric branch conditions [12], which means the $2 \times 2$ impedance model truncated under the symmetric conditions can still be used without losing accuracy. Despite that, the presented stability analyses concerning medium and strong asymmetry are still meaningful in justifying the integrity of the proposed model reduction method, i.e., the global applicability. What's more, extreme unbalanced branch conditions are very common in the distribution system. For example, Ref [9] reported an asymmetric MMC case with Aphase inductance nearly 3 times smaller than the B-/C-phase, which happened in a railway electrical system.

2) Asymmetric AC-grid Conditions

Case 4: As shown in Fig. 9(a), the $\boldsymbol{L}_{d q \pm}$ can predict the correct stability conclusion, i.e., the marginally stable state with $\boldsymbol{R}_{\mathrm{g} x}=6 \Omega$ and the marginally unstable state with $\boldsymbol{R}_{\mathrm{g} x}=5 \Omega$, which conforms with the time-domain simulations. Here, it should be noted that although the $\lambda_{1}$ (previous state and latter 

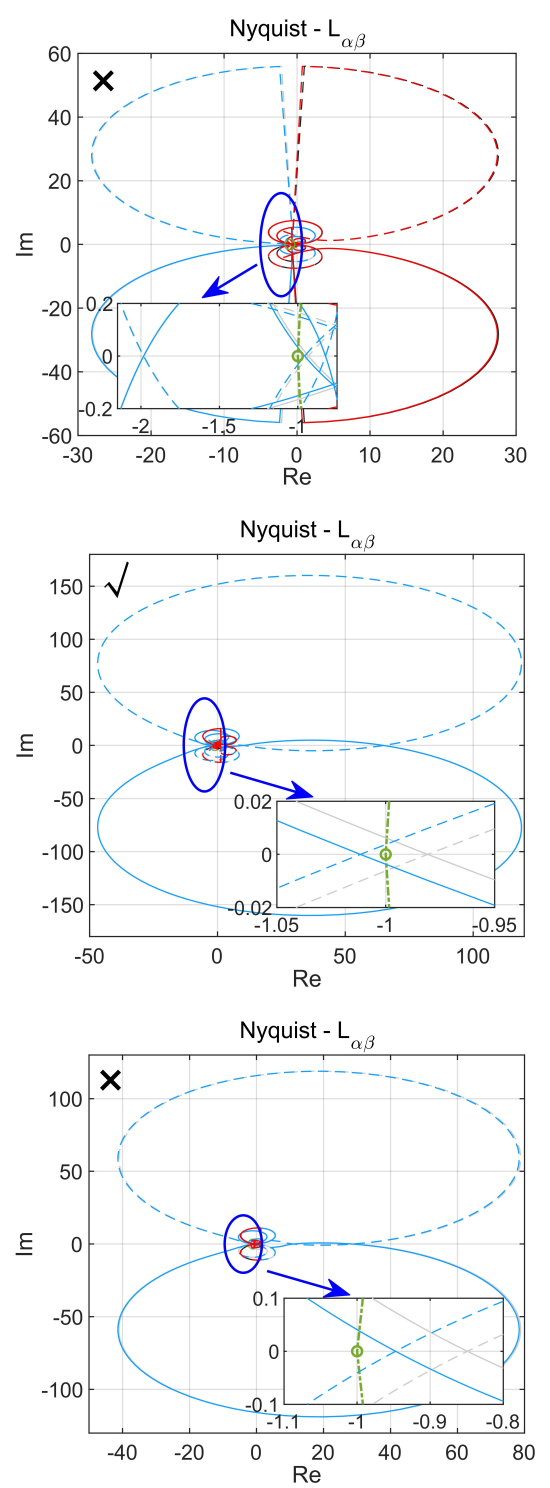

Previous State

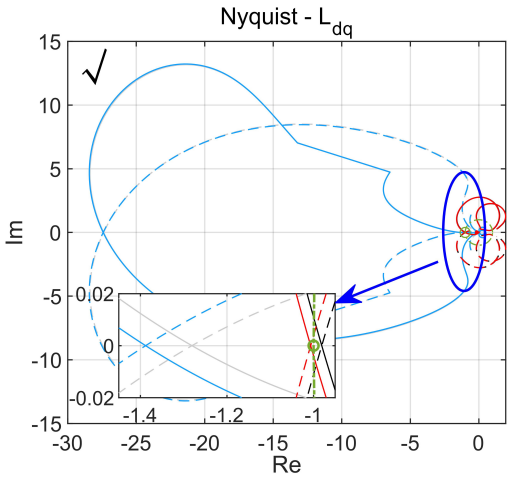

(a)

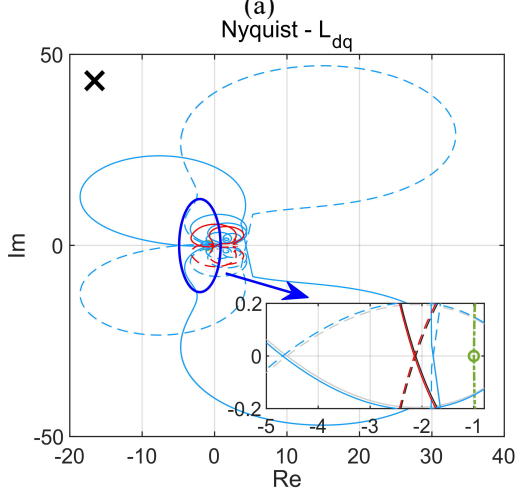

(b)

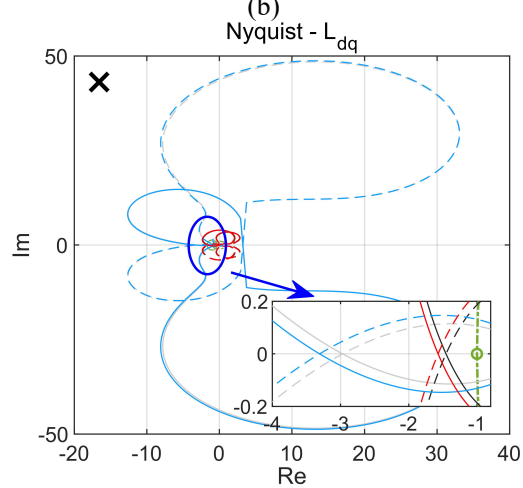

(c)
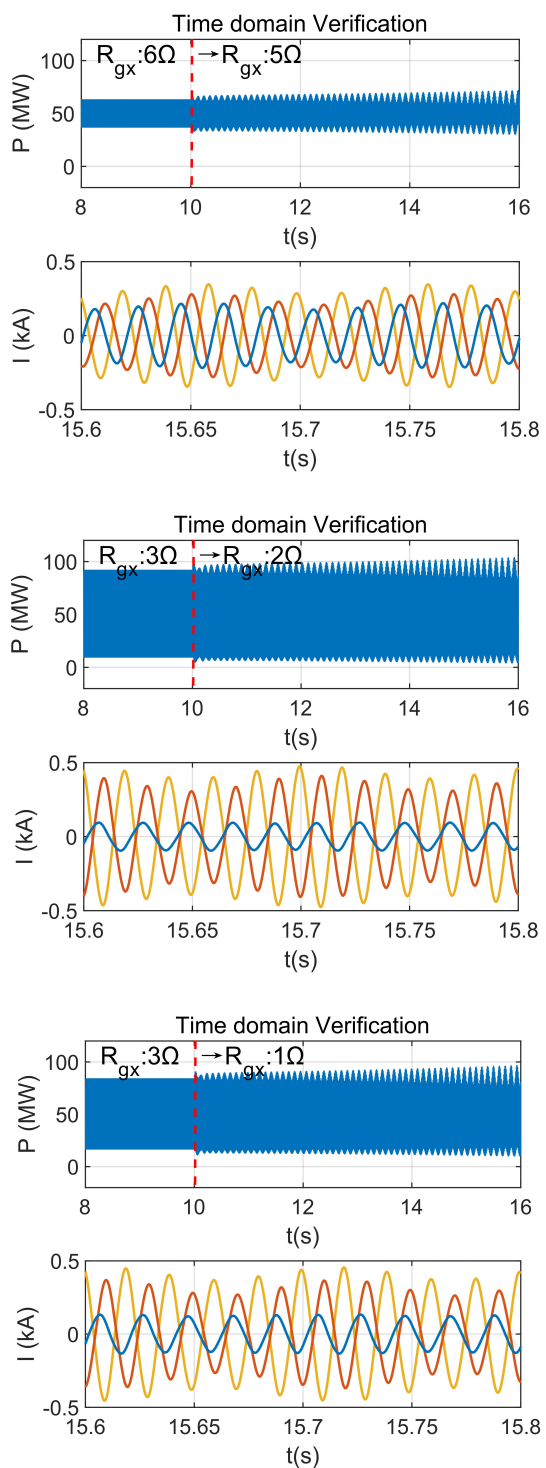

$\operatorname{conj}\left(\lambda_{1}\right)-\lambda_{2}----\operatorname{conj}\left(\lambda_{2}\right)$

$(-1,0) \ldots$ unit cycle

Latter State

Fig. 9. Theoretical stability analysis and time-domain verifications, (a) Case 4; (b) Case 5; (c) Case 6.

state) cross the left half real axis, they do not surround the (-1, j0) which can be observed more clearly from the partial enlarged subfigure in Fig. 9(a). A counterexample using $\boldsymbol{L}_{\alpha \beta \pm}$ is also given in Fig. 9(a), where a pessimistic stability conclusion is obtained, i.e., the unstable state with $\boldsymbol{R}_{\mathrm{g} x}=6 \Omega$ and $5 \Omega$. Now, it is again verified that the $2 \times 2$ impedance in $d$ $q$ domain can predict the stability conclusion accurately under the Weak Asymmetry.

Case 5: As shown in Fig. 9(b), the $\boldsymbol{L}_{\alpha \beta \pm}$ can predict the correct stability conclusion, i.e., the marginally stable state with $\boldsymbol{R}_{\mathrm{g} x}=3 \Omega$ and the marginally unstable state with $\boldsymbol{R}_{\mathrm{g} x}=2 \Omega$, which conforms with the time-domain simulations. A counterexample using $\boldsymbol{L}_{d q \pm}$ is also given in Fig. 9(b), where an extremely pessimistic conclusion is obtained, i.e., the unstable state with $\boldsymbol{R}_{\mathrm{g} x}=3 \Omega$ and $2 \Omega$. Now, it is again verified that the 2 $\times 2$ impedance in $\alpha-\beta$ domain can predict the stability conclusion accurately under the Strong Asymmetry.
Case 6: As shown in Fig. 9(c), compared with the timedomain results, neither the $\boldsymbol{L}_{\alpha \beta \pm}$ nor the $\boldsymbol{L}_{d q \pm}$ can give an accurate stability conclusion. The obtained stability result is more pessimistic using $\boldsymbol{L}_{d q \pm}$, while more optimistic using $\boldsymbol{L}_{\alpha \beta \pm}$.

Based on the above analysis, similar conclusions can be drawn from asymmetric ac-grid cases, which further verify the global applicability of the proposed method. In conclusion, for most of asymmetric cases, the accurate stability conclusion can be obtained using the reduced-order model $(2 \times 2)$ either in $\alpha-\beta$ domain or $d-q$ domain, depending on which one can better satisfy the prerequisites of the Lemma $\mathbf{1}$ (i.e., BDD system).

It should be noted that the above two asymmetric scenarios, i.e., unbalanced branch conditions and unbalanced ac-grid conditions, are only served as an example to demonstrate how the proposed method is implemented, which can also be applied in other asymmetric cases as long as the BDD characteristics of the studied case are properly evaluated. 


\section{CONCLUSION}

This paper presented a block diagonal dominance (BDD)based model reduction method aiming for simpler frequencydomain/impedance-based stability analysis.

Application of this method in the model reduction and stability of the MMC is shown in detail. In which, the applied permutation matrix for the applicability improvement of BDD characteristics turns out to be the rotation matrix from $\alpha-\beta$ to $d$ $q$ domain. Moreover, it is demonstrated that by using this permutation matrix, a $2 \times 2$ reduced-order impedance model of the MMC can be acquired either in $\alpha-\beta$ or $d-q$ domain. Specifically, the impedance model in $\alpha-\beta$ domain is more preferred for the strong asymmetry, while the impedance model in $d-q$ domain exhibits better BDD property for the weak asymmetry. This useful finding is justified via the case studies considering different asymmetry degrees, including the typical asymmetric MMC scenario and ac-grid scenario.

Although the grid-connected MMC system is used as an example to demonstrate the proposed BDD-based model reduction method, it is worth of mentioning that by following a similar principle and BDD evaluation procedure, the proposed method can be applied to other asymmetric cases. Therefore, it shows potential for being promoted as a systematic tool for simplifying the frequency-domain/impedance analysis, particularly for asymmetric cases. This is worth of being explored in future works.

\section{APPENDIX A}

The $\boldsymbol{N}_{\text {hss }}$ is defined as:

$$
N_{\text {hss }}=\left[\begin{array}{llll}
N_{\mathrm{p}} & & & \\
& N_{\mathrm{p}} & & \\
& & N_{\mathrm{p}} & \\
& & & N_{\mathrm{p}}
\end{array}\right]
$$

where $\quad \boldsymbol{N}_{\mathrm{p}}=\operatorname{blkdiag}\left(\boldsymbol{N}_{\mathrm{p} a}, \boldsymbol{N}_{\mathrm{p} b}, \boldsymbol{N}_{\mathrm{p} c}\right)$ $\boldsymbol{N}_{\mathrm{p} a}=\boldsymbol{N}_{\mathrm{p} b}=\boldsymbol{N}_{\mathrm{p} c}=\operatorname{diag}\left(s-j h \omega_{1}, \ldots, s, \ldots, s+j h \omega_{1}\right)$.

The $a_{\mathrm{hss}}$ is defined as:

$$
\boldsymbol{a}_{\mathrm{hss}}=\left[\begin{array}{cccc}
-\frac{\boldsymbol{R}_{\mathrm{arm} x}}{\boldsymbol{L}_{\mathrm{arm} x}} & -\frac{\boldsymbol{M}_{\mathrm{u} x}^{\mathrm{hss}}}{2 \boldsymbol{L}_{\mathrm{arm} x}} & -\frac{\boldsymbol{M}_{\mathrm{l} x}^{\mathrm{hss}}}{2 \boldsymbol{L}_{\mathrm{arm} x}} & \mathbf{0} \\
\frac{\boldsymbol{M}_{\mathrm{u} x}^{\mathrm{hss}}}{\boldsymbol{C}_{\mathrm{arm} x}} & \mathbf{0} & \mathbf{0} & \frac{\boldsymbol{M}_{\mathrm{u} x}^{\mathrm{hss}}}{2 \boldsymbol{C}_{\mathrm{arm} x}} \\
\frac{\boldsymbol{M}_{\mathrm{l} \boldsymbol{x}}^{\mathrm{hss}}}{\boldsymbol{C}_{\mathrm{arm} x}} & \mathbf{0} & \mathbf{0} & -\frac{\boldsymbol{M}_{\mathrm{l} \boldsymbol{x}}^{\mathrm{hss}}}{2 \boldsymbol{C}_{\mathrm{arm} x}} \\
\mathbf{0} & -\frac{\boldsymbol{M}_{\mathrm{u} x}^{\mathrm{hss}}}{\boldsymbol{L}_{\mathrm{arm} x}} & \frac{\boldsymbol{M}_{\mathrm{l} x}^{\mathrm{hss}}}{\boldsymbol{L}_{\mathrm{arm} x}} & -\frac{\boldsymbol{R}_{\mathrm{arm} x}}{\boldsymbol{L}_{\mathrm{arm} x}}
\end{array}\right]
$$

The $m_{\mathrm{hss}}$ is defined as:

The $b_{\mathrm{hss}}$ is defined as:

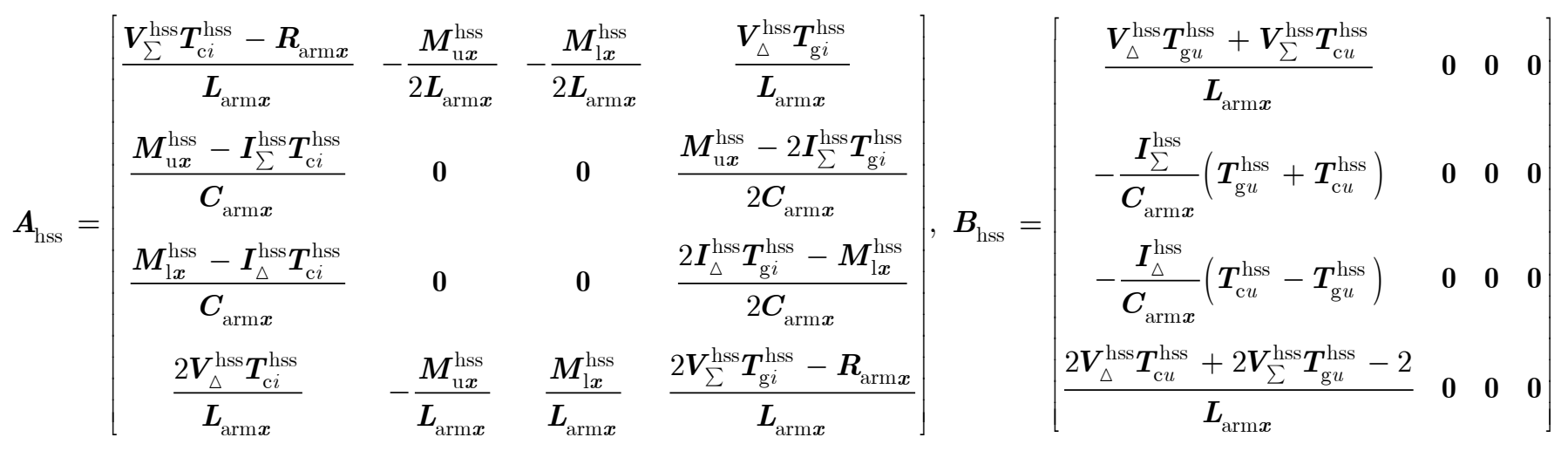

The definitions of the $\boldsymbol{A}_{\mathrm{hss}}$ and $\boldsymbol{B}_{\mathrm{hss}}$ are given in (A5) (at the bottom of this page).

The above used $\boldsymbol{V}_{\Sigma}^{\mathrm{hss}}, \boldsymbol{V}_{\Delta}^{\mathrm{hss}}, \boldsymbol{I}_{\Sigma}^{\mathrm{hss}}, \boldsymbol{I}_{\Delta}^{\mathrm{hss}}$ are defined as:

$$
\begin{gathered}
\boldsymbol{V}_{\Sigma}^{\mathrm{hss}}=\frac{\boldsymbol{V}_{\mathrm{cu} \boldsymbol{x}}^{\mathrm{hss}}+\boldsymbol{V}_{\mathrm{cl} \boldsymbol{x}}^{\mathrm{hss}}}{2 V_{\mathrm{dc}}}, \boldsymbol{V}_{\Delta}^{\mathrm{hss}}=\frac{\boldsymbol{V}_{\mathrm{cu} \boldsymbol{x}}^{\mathrm{hss}}-\boldsymbol{V}_{\mathrm{cl} \boldsymbol{x}}^{\mathrm{hss}}}{2 V_{\mathrm{dc}}} \\
\boldsymbol{I}_{\Sigma}^{\mathrm{hss}}=\frac{2 \boldsymbol{I}_{\mathrm{c} \boldsymbol{x}}^{\mathrm{hss}}+\boldsymbol{I}_{\mathrm{g} \boldsymbol{x}}^{\mathrm{hs}}}{2 V_{\mathrm{dc}}}, \boldsymbol{I}_{\Delta}^{\mathrm{hss}}=\frac{2 \boldsymbol{I}_{\mathrm{c} \boldsymbol{x}}^{\mathrm{hs}}-\boldsymbol{I}_{\mathrm{g} \boldsymbol{x}}^{\mathrm{hss}}}{2 V_{\mathrm{dc}}}
\end{gathered}
$$




\section{APPENDIX B}

By definition, harmonics result from periodic steady state operating conditions, and thus their prediction should be formulated in terms of (harmonic) phasors, i.e., in the frequency couplings. Various couplings generated in the system can be collectively referred as (harmonic) frequency couplings. In practical application, a decoupled model is more preferred. However, for the commonly used $a b c$ frame and $d-q$ frame, a model without coupling terms is hardly to be obtained due to the inherent inter-phase or $d-q$ couplings. Therefore, the complex vector frame is widely applied, where two most representative frames are sequence domain $(\alpha \pm j \beta)$ and modified sequence domain $(d \pm j q)$. It is noted that by selecting the appropriate complex vector frame, the off-diagonal terms can be rotated to the principal diagonal.

Sequence coupling [8]: the coupling across the $\alpha+j \beta$ (positive sequence) and $\alpha-j \beta$ (negative sequence) at the same frequency. The strength of such coupling is positively correlated with the three-phase asymmetry. If the system is three-phase symmetric, the sequence coupling should be zero.

Mirror Frequency coupling [19]: the coupling across the ' $s$ ' and ' $s-2 j \omega_{1}$ '. The strength of such coupling is positively correlated with the control asymmetry (e.g., PLL, outer loops, etc.). If the control scheme is symmetric, the mirror frequency coupling should be zero and the system is called 'mirror frequency decoupled (MFD)'.

\section{REFERENCES}

[1] A. Lesnicar and R. Marquardt, "An innovative modular multilevel converter topology suitable for a wide power range," 2003 IEEE Bologna Power Tech Conference Proceedings, Bologna, Italy, 2003, pp. 6 pp. Vol.3-

[2] Kamran Sharifabadi; Lennart Harnefors; Hans-Peter Nee; Staffan Norrga; Remus Teodorescu, "Introduction," in Design, Control, and Application of Modular Multilevel Converters for HVDC Transmission Systems, IEEE, 2016, pp.1-6.

[3] X. Wang and F. Blaabjerg, "Harmonic Stability in Power ElectronicBased Power Systems: Concept, Modeling, and Analysis," in IEEE Transactions on Smart Grid, vol. 10, no. 3, pp. 2858-2870, May 2019.

[4] A. Jamshidifar and D. Jovcic, "Small-Signal Dynamic DQ Model of Modular Multilevel Converter for System Studies," in IEEE Transactions on Power Delivery, vol. 31, no. 1, pp. 191-199, Feb. 2016.

[5] G. Bergna-Diaz, J. A. Suul and S. D'Arco, "Energy-Based State-Space Representation of Modular Multilevel Converters with a Constant Equilibrium Point in Steady-State Operation," in IEEE Transactions on Power Electronics, vol. 33, no. 6, pp. 4832-4851, June 2018.

[6] Ö. C. Sakinci and J. Beerten, "Generalized Dynamic Phasor Modeling of the MMC for Small-Signal Stability Analysis," in IEEE Transactions on Power Delivery, vol. 34, no. 3, pp. 991-1000, June 2019.

[7] J. Lyu, X. Zhang, X. Cai and M. Molinas, "Harmonic State-Space Based Small-Signal Impedance Modeling of a Modular Multilevel Converter With Consideration of Internal Harmonic Dynamics," in IEEE Transactions on Power Electronics, vol. 34, no. 3, pp. 2134-2148, March 2019

[8] J. Sun and H. Liu, "Sequence Impedance Modeling of Modular Multilevel Converters," in IEEE Journal of Emerging and Selected Topics in Power Electronics, vol. 5, no. 4, pp. 1427-1443, Dec. 2017.

[9] M. Lei et al., "Full Degree of Freedom Based Control Scheme of the Single-Phase Direct AC-AC Modular Multilevel Converter for Railway Power Conditioning Under Asymmetric Branch Conditions," in IEEE Transactions on Industrial Electronics, vol. 67, no. 3, pp. 1671-1683, March 2020
[10] Y. Zhou, D. Jiang, J. Guo, P. Hu and Y. Liang, "Analysis and Control of Modular Multilevel Converters Under Unbalanced Conditions," in IEEE Transactions on Power Delivery, vol. 28, no. 4, pp. 1986-1995, Oct. 2013.

[11] X. Shi, Z. Wang, B. Liu, Y. Li, L. M. Tolbert and F. Wang, "Steady-State Modeling of Modular Multilevel Converter Under Unbalanced Grid Conditions," in IEEE Transactions on Power Electronics, vol. 32, no. 9, pp. 7306-7324, Sept. 2017

[12] F. Deng, Q. Heng, C. Liu and Q. Wang, "Circulating current suppression method for MMC-HVDC systems with asymmetric arm impedance," in CSEE Journal of Power and Energy Systems.

[13] C. Guo, J. Yang and C. Zhao, "Investigation of Small-Signal Dynamics of Modular Multilevel Converter Under Unbalanced Grid Conditions," in IEEE Transactions on Industrial Electronics, vol. 66, no. 3, pp. 22692279, March 2019.

[14] J. Zhu, J. Hu, S. Wang and M. Wan, "Small-Signal Modeling and Analysis of MMC under Unbalanced Grid Conditions Based on Linear TimePeriodic (LTP) Method," in IEEE Transactions on Power Delivery.

[15] H. Wu, X. Wang and Ł. Kocewiak, "Impedance-Based Stability Analysis of Voltage-Controlled MMCs Feeding Linear AC Systems," in IEEE Journal of Emerging and Selected Topics in Power Electronics, early access.

[16] H. Zong, C. Zhang, J. Lyu, X. Cai, M. Molinas and F. Rao, "Generalized MIMO Sequence Impedance Modeling and Stability Analysis of MMCHVDC With Wind Farm Considering Frequency Couplings," IEEE Access, vol. 8, pp. 55602-55618, 2020.

[17] Y. Chen, L. Xu, A. Egea-Álvarez, B. Marshall, M. Rahman and O. D. Adeuyi, "MMC Impedance Modelling and Interaction of Converters in Close Proximity," in IEEE Journal of Emerging and Selected Topics in Power Electronics, early access.

[18] A. MacFarlane, "The development of frequency-response methods in automatic control [Perspectives]," in IEEE Transactions on Automatic Control, vol. 24, no. 2, pp. 250-265, April 1979.

[19] C. Zhang, X. Cai, A. Rygg and M. Molinas, "Sequence Domain SISO Equivalent Models of a Grid-Tied Voltage Source Converter System for Small-Signal Stability Analysis," in IEEE Transactions on Energy Conversion, vol. 33, no. 2, pp. 741-749, June 2018

[20] Z. Xu et al., "A Complete HSS Based Impedance Model of MMC Considering Grid Impedance Coupling," in IEEE Transactions on Power Electronics.

[21] H. H. Rosenbrock, "Design of multivariable control systems using the inverse Nyquist array," in Proceedings of the Institution of Electrical Engineers, vol. 116, no. 11, pp. 1929-1936, November 1969.

[22] W.H. Bennett, J.S. Baras, Block Diagonal Dominance and Design of Decentralized Compensators, IFAC Proceedings Volumes, Volume 13, Issue 6, 1980, Pages 93-101.

[23] D. I. X. Limebeer, "The application of generalized diagonal dominance to linear system stability theory." Int. J. Contr., vol. 36. pp.185-212. 1982.

[24] C. Zhang, M. Molinas, A. Rygg, J. Lyu and X. Cai, "Harmonic TransferFunction-Based Impedance Modeling of a Three-Phase VSC for Asymmetric AC Grid Stability Analysis," in IEEE Transactions on Power Electronics, vol. 34, no. 12, pp. 12552-12566, Dec. 2019.

[25] Y. Li, Y. Gu and T. C. Green, "Interpreting Frame Transformations in AC Systems as Diagonalization of Harmonic Transfer Functions," in IEEE Transactions on Circuits and Systems I: Regular Papers.

[26] F. Liu, J. Liu, H. Zhang and D. Xue, "Stability Issues of \$Z + Z\$ Type Cascade System in Hybrid Energy Storage System (HESS)," in IEEE Transactions on Power Electronics, vol. 29, no. 11, pp. 5846-5859, Nov. 2014

[27] L. Chen, H. Nian and Y. Xu, "Complex transfer function-based sequence domain impedance model of doubly fed induction generator," in IET Renewable Power Generation, vol. 13, no. 1, pp. 67-77, 712019.

[28] Y. Ren, R. Duan, L. Chen, W. Huang, C. Wu and Y. Min, "Stability Assessment of Grid-connected Converter System Based on Impedance Model and Gershgorin Theorem," in IEEE Transactions on Energy Conversion.

[29] L. Harnefors, X. Wang, S. Chou, M. Bongiorno, M. Hinkkanen and M. Routimo, "Asymmetric Complex-Vector Models With Application to VSC-Grid Interaction," in IEEE Journal of Emerging and Selected Topics in Power Electronics, vol. 8, no. 2, pp. 1911-1921, June 2020. 


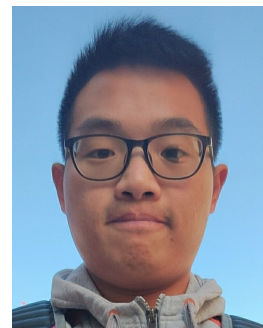

HAOXIANG ZONG (S'18) received the B.Eng. degree in electrical engineering from the Tianjin University, Tianjin, China, in 2017. He is currently pursuing the Ph.D. degree in electrical engineering with Shanghai Jiao Tong University, Shanghai, China. From 2019 to 2021, he has been a Ph.D. Visiting Scholar at the Department of Engineering Cybernetics, Norwegian University of Science and Technology, Trondheim, Norway. His current research interest is modeling and stability analysis of wind farm systems and MMCs.

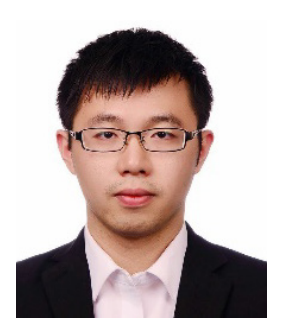

CHEN ZHANG (M'20) received the B.Eng. degree from the China University of Mining and Technology, China, and the $\mathrm{Ph} . D$. from Shanghai Jiao Tong University, China, in 2011 and 2018 respectively. He was a Postdoctoral Research Fellow at the Department of Engineering Cybernetics of NTNU, from March 2018 to October 2020. Currently, he is a postdoctoral researcher with the Department of Electrical Engineering, Technical University of Denmark, Lyngby, Denmark. His research interest is modeling and stability analysis of VSC-based energy conversion systems, where the aim is to reveal the fundamental dynamics and stability mechanisms of renewable energies with VSCs as the grid interface.

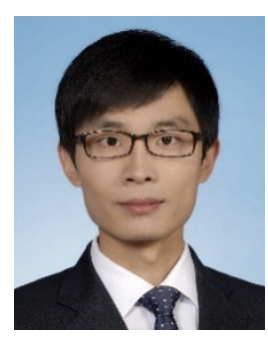

JING LYU (S'14-M'16-SM'20) received the B.Eng. degree from China University of Mining and Technology, Jiangsu, China, in 2009, the M.Eng. and Ph.D. degrees from Shanghai Jiao Tong University, Shanghai, China, in 2011 and 2016, respectively, all in electrical engineering. He was a Postdoctoral Research Fellow with the Department of Engineering Cybernetics, Norwegian University of Science and Technology, Trondheim, Norway, from 2016 to 2017. Since 2018, he has been with the Department of Electrical Engineering, Shanghai Jiao Tong University, where he is now a Tenure-Track Associate Professor. His main research interests include dynamic stability of MMC-based HVDC connected wind farms/PV plants, modeling and control of modular multilevel converter, wind power converters, and applications of artificial intelligence in power electronic systems.

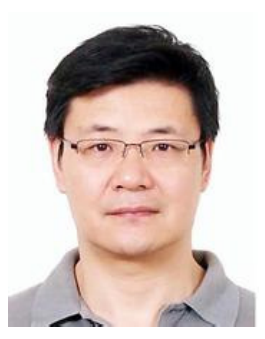

XU CAI received the B.Eng. degree in electrical engineering from Southeast University, Nanjing, China, in 1983, the M.Eng. and Ph.D. degrees from China University of Mining and Technology, Jiangsu, China, in 1988 and 2000, respectively, both in electrical engineering. He was with the Department of Electrical Engineering, China University of Mining and Technology, as an Associate Professor from 1989 to 2001. He was the Vice Director of the State Energy Smart Grid R\&D Center, Shanghai, China, from 2010 to 2013. He has been with Shanghai Jiao Tong University, Shanghai, as a Professor since 2002, where he has also been the Director of the Wind Power Research Center since 2008. His current research interests include power electronics and renewable energy exploitation and utilization, including wind power converters, wind turbine control system, large power battery storage systems, clustering of wind farms and its control system, and grid integration.

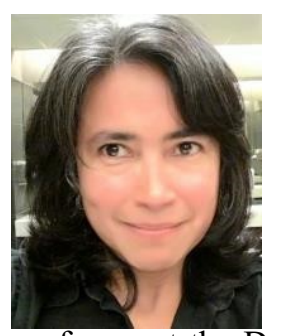

MARTA MOLINAS (M'94) received the Doctor of Engineering degree from the Tokyo Institute of Technology, Tokyo, Japan, in 2000. She was a Guest Researcher with the University of Padova, Padova, Italy, during 1998 and JSPS Fellow at AIST Tsukuba, Japan from 2008 to 2009. From 2008-2014 she has been professor at the Department of Electric Power Engineering at NTNU and since 2014 Professor at the Department of Engineering Cybernetics, NTNU. In the period 2013-2014 she has been visiting scholar at Columbia University Earth Institute Sustainable Engineering Lab and Bhutan Prime Minister Fellow working on the renewable energy policy for the Kingdom. She is currently on her sabbatical year at the Innovation Medical Research Institute of Tsukuba University. Her research interests include stability of power electronics systems, harmonics and non-stationary signals from the human and the machine. She is Editor for the IEEE Journal JESTPE and the IEEE Trans. on Energy Conversion, Ass. Editor for the IEEE PELS and the IEEE TIE. 